\title{
Identifying the Optimal Role for Pharmacists in Care Transitions: A Systematic Review
}

\author{
Hendrik T. Ensing, PharmD; Clementine C. M. Stuijt, PharmD; Bart J. F. van den Bemt, PharmD, PhD; \\ Ad A. van Dooren, PharmD, PhD; Fatma Karapinar-Çarkit, PharmD, PhD; Ellen S. Koster, PhD;
} and Marcel L. Bouvy, PharmD, PhD

\begin{abstract}
BACKGROUND: A transition from one health care setting to another increases the risk of medication errors. Several strategies have been applied to improve care transitions and reduce adverse clinical outcomes. Pharmacist intervention during and after hospitalization has been frequently studied and show a variable effect on these outcomes.
\end{abstract}

OBJECTIVE: To identify the components of pharmacist intervention that improve clinical outcomes during care transitions.

METHODS: MEDLINE, EMBASE, International Pharmaceutical Abstracts, and Web of Science databases were searched for randomized controlled trials (RCTs) that studied pharmacist intervention with regard to hospitalization. Two reviewers independently screened all references published from inception to November 2014, extracted data, and assessed risk of bias.

RESULTS: A total of 30 studies met the inclusion criteria. A model was created to categorize and cluster components of pharmacist intervention. The average number of components deployed, stages of hospitalization covered, and intervention targets were equally distributed between effective and ineffective studies. A best evidence synthesis of 15 studies revealed strong evidence for a clinical medication review in multifaceted programs (5 effective vs. 0 ineffective studies). Conflicting evidence was found for an isolated postdischarge intervention, admission medication reconciliation, combining postdischarge interventions with in-hospital interventions, and covering of multiple stages. Closely collaborating with other health care providers enhanced the effectiveness.

CONCLUSIONS: Although there is a need for well-designed and wellreported RCTs, the study heterogeneity enabled a best evidence synthesis to elucidate effective components of pharmacist intervention. In isolated postdischarge intervention programs, evidence tends towards collaborating with nurses and tailoring to individual patient needs. In multifaceted intervention programs, performing medication reconciliation alone is insufficient in reducing postdischarge clinical outcomes and should be combined with active patient counseling and a clinical medication review. Furthermore, close collaboration between pharmacists and physicians is beneficial. Finally, it is important to secure continuity of care by integrating pharmacists in these multifaceted programs across health care settings. Ultimately, pharmacists need to know patient clinical background and previous hospital experience.

J Manag Care Spec Pharm. 2015;21(8):614-38

Copyright $\odot 2015$, Academy of Managed Care Pharmacy. All rights reserved.

\section{What is already known about this subject}

Transitions between health care settings increase the risk of medication errors, which can result in adverse drug events, prolonged hospital stay, early readmissions, and use of other health care resources.

Pharmacist intervention during and after hospitalization have been frequently studied, albeit with varied effects on clinical outcomes.

Several systematic reviews have been performed studying care transition programs, although none have done so by separating pharmacist intervention components from continuity of care programs

\section{What this study adds}

Our model systematically categorized components of pharmacist intervention in care transition programs. Study heterogeneity enabled a best evidence synthesis to elucidate effective components.

This review revealed that multifaceted programs should combine medication reconciliation with active patient counseling and a clinical medication review. Care continuity can be secured by integrating pharmacists across settings and providing them with patients' clinical background.

Collaborating with other health care professionals is crucial to increase the effectiveness of pharmacist intervention.

A transition from one health care setting to another increases the risk of medication errors. Medication errors have been particularly attributed to poor communication or loss of important information. ${ }^{1}$ These errors can result in clinically relevant outcomes such as adverse drug events (ADEs), increased duration of hospital stay, early readmissions after discharge, and use of other health care resources. ${ }^{2}$ Although ADEs generally are the most invasive type of drug-related problems (DRPs), other DRPs may also result in patient harm, which then results in unplanned hospital readmissions. ${ }^{3}$

Numerous strategies have been applied to reduce the number of ADEs and (drug-related) readmissions by involving various health care professionals such as nurses and pharmacists. ${ }^{4-7}$ Because of the likely link between DRPs and adverse 
clinical outcomes, pharmacists may be the preferred health care provider to intervene and reduce the risks involved in care transitions, a view endorsed by 2 Institute of Medicine reports. ${ }^{8,9}$ Pharmacist intervention during and after hospitalization has been studied, albeit with varying effects on clinical outcomes. Some studies have shown significant reduction in drug-related readmissions, whereas others have shown improved surrogate outcomes (e.g., medication appropriateness or knowledge) but lacked significant impact on readmissions or had no effect at all. ${ }^{10-13}$ Other studies have revealed a significant reduction in readmission rates but did not use a randomized study design..$^{14,15}$

Several systematic reviews have studied care transition programs. ${ }^{2,16-29}$ However, these reviews focused either on a specific intervention component (e.g., hospital-based medication reconciliation); an isolated health care setting (e.g., an inpatient care setting); a specific high-risk population (e.g., heart failure patients); included only 1 outcome (e.g., readmissions); or did not specifically target pharmacist intervention. Finally, most reviews lacked an extensive description of the intervention components deployed in the included studies.

The purpose of this systematic review was to focus specifically on unraveling the components of pharmacist intervention from continuity of care programs that improved clinical outcomes.

\section{Methods}

\section{Search Strategy}

This systematic review was conducted in accordance with the Preferred Reporting Items for Systematic Reviews and MetaAnalyses (PRISMA) guidelines (Appendix A, available in online article). ${ }^{30}$ Three electronic databases were searched (MEDLINE, EMBASE, and International Pharmaceutical Abstracts [IPA]) from inception to November 2014. Randomized controlled trials (RCTs) investigating interventions with regard to adult hospitalization and discharge with a proactive role for a pharmacist of any type (e.g., hospital, community, clinical) were identified (Appendix B, available in online article). The search strategy was designed in MEDLINE using the following medical subject headings and text words: patient education, counseling, medication therapy management, medication errors/prevention and control, medication reconciliation, continuity of patient care, patient care planning, aftercare, house calls, and drug utilization review. Synonymous terms combined with words for hospital admission and pharmacist profession were also used (see Appendix C for detailed search terms, available in online article). Only studies in English were included. The search strategy was further refined and validated by indexing known relevant articles. For EMBASE and IPA, search terms were adapted according to the capabilities of these particular databases. Reference lists of all included trials, previous systematic reviews, and the citation indexing service Web of Science were checked manually for additional relevant publications.

\section{Review Process}

The reference management software RefWorks was used to manage all citations (ProQuest LLC, Ann Arbor, MI). First, each reference title was screened independently by 2 reviewers (authors Ensing and Stuijt) for eligibility against the agreed inclusion and exclusion criteria (Appendix B). Next, all included abstracts were screened. Finally, the resulting fulltext copies of all studies considered to be of potential relevance were retrieved and screened similarly. Inter-rater agreement was calculated, and disagreement between the reviewers was resolved through discussion.

\section{Data Collection}

Data from included trials were extracted into MS Excel 2007 (Microsoft Corporation, Redmond, WA) by 1 of the 2 initial reviewers and independently checked by a second reviewer from among the authors of this study. The following characteristics of each study were retrieved: general information (first author, year of publication); study design (multicenter or single center, hospital and ward type); patient characteristics (sample size, gender, age, number of medications, health state); method (inclusion and exclusion criteria, usual care, pharmacist intervention components, coinvolved health care provider [HCP]); study outcomes; and conclusions. Appendix D (available in online article) contains a complete list of extracted parameters.

\section{Quality Assessment of Individual Studies}

The methodological quality of the studies was independently assessed by the 2 reviewers according to the Cochrane risk of bias tool. ${ }^{31}$ This is a domain-based evaluation in which critical assessments are made over 7 separate domains: random sequence generation, allocation concealment, blinding of participants and personnel, blinding of outcome assessors, incomplete outcome data, selective reporting, and other sources of bias. The nature of the studied interventions implicated unblinded personnel and participants, resulting in a high risk of performance bias for all included studies and a maximum score of 6 bias-free domains. Other domains were scored as high, low, or unclear risk of bias. Disagreement was resolved through discussion.

\section{Data Synthesis and Analysis}

The included studies were heterogeneous regarding the intervention components, included populations, coinvolved HCPs, and outcomes. Therefore, statistical aggregation of findings was deemed inappropriate, and a qualitative analysis was performed. ${ }^{32,33}$ The following steps were undertaken to systematically categorize the results:

1. Only pharmacist intervention components reported in the original manuscript were used.

2. All pharmacist intervention components were screened and categorized independently by the 2 author/reviewers, 
creating a pharmacist intervention model (Table 1) covering all components.

3. The 15 intervention components included in the pharmacist intervention model were structured by applying 3 types of clustering (Table 1):

a. Target: patient-aimed or HCP-aimed interventions.

b. Nature: pharmacist professional care or administrative interventions. Professional care interventions included all services using pharmacists' skills and knowledge for an active role in patient health care. Administrative interventions comprised providing and handling of documents, for example.

c. Stage: intervention performed at admission, during admission, at discharge, postdischarge, or stage-independent.

4. All outcomes were extracted, and-if not supplied by the respective study authors-effectiveness rates were calculated. Studies were categorized as "effective" in cases where at least 1 of the predefined outcomes was statistically significant (inclusion criteria, Appendix B). In case of a mixed effect (e.g., a significant increase in ADEs and a significant decrease in emergency department visits), the following priority of clinical relevance was applied: (a) mortality, (b) readmissions, (c) emergency department visits, and (d) ADEs, with the latter being the least relevant. Statistical significance was set at $P<0.05$.

5. Average numbers of intervention components according to the clusters previously mentioned were compared between effective and ineffective studies. Data were checked for normality, and either an independent t-test or Mann-Whitney $\mathrm{U}$ test was performed to detect significant differences $(P<0.05)$.

Finally, a best evidence synthesis was conducted according to the framework proposed by Treadwell et al. (2012). ${ }^{34}$ Since our review included only RCTs, a stringent threshold in methodological quality was used to define the "best evidence set" by including studies with 5 or more bias-free domains. To attribute various levels of evidence to the effectiveness of the pharmacist intervention components, all reported study parameters ([combinations of] interventions, intervention stages, coinvolved HCPs, pharmacist type, setting characteristics) were taken into account. Evidence levels were based on van Tulder et al. (2003) $)^{35}$ and are as follows: (a) Strong-consistent findings among multiple high quality RCTs; (b) Moderate_findings in 1 high quality RCT; and (c) Conflicting-inconsistent findings among multiple high quality RCTs. Since only RCTs were included, the levels Limited evidence and No evidence were not applicable.
Results

\section{Study Selection}

The searches identified 3,084 records, which resulted in 2,619 nonduplicate items. Thirty papers met the inclusion criteria and were included in this systematic review (Figure 1). ${ }^{6,10-13,36-60}$ The observed similarity between the 2 author/reviewers for full-text screening was 94\% (inter-rater agreement $\kappa=0.87$, Appendix E, available in online article), and all disagreements were resolved through discussion. Reasons for exclusion at this stage are given in Appendix F (available in online article).

\section{Study Characteristics}

The 30 included studies covered all Western continents: North America $(n=15)$, Europe $(n=10)$, and Australia $(n=5)$. Just over half of the studies $(\mathrm{n}=17)$ were conducted in an academic, teaching, or tertiary referral hospital (Table 2). Eleven studies implemented a hospital-wide intervention program, whereas others focused on patients in specific wards (mainly internal or general medicine, $\mathrm{n}=11$ ). The number of included patients varied considerably among the studies (range $=34-936$ ). Subjects in both arms of these studies were generally well matched. Eighteen studies included patients with predefined health conditions or other high-risk factors. These were mainly chronic heart failure $(n=8)$ or acute hospital admission ( $n=5$; Table 2$)$.

\section{Quality Assessment of Individual Studies}

Of the total 180 domains, $19 \%(n=34)$ were scored differently by the 2 reviewers and resolved through discussion. Eight studies scored low risk for bias in all 6 domains (Appendix $G$, available in online article). ${ }^{13,39-41,48,54,55,59}$ Of the items in the selection bias, detection bias, attrition bias, and reporting bias domains, $11 \%$ could not be assessed due to insufficient data in the original studies. Five studies were considered at high risk for other bias; all had contamination bias because the same pharmacist took care of the intervention and control groups. ${ }^{10,11,45,56,58}$ Finally, there was an unclear risk of other bias with an unclear effect on study outcomes in 10 studies: possible contamination bias, ${ }^{12,44,49,50}$ possible compliance bias, ${ }^{36,42,57}$ possible recall bias of participants, ${ }^{51,60}$ and baseline differences with lack of power for adequate conclusions. ${ }^{37}$

\section{Results of Individual Studies: Pharmacist Interventions and Outcome Measurements}

The overall number of intervention components for a specific outcome is presented in Appendix H (available in online article). This cross-tab illustrates, together with the heterogeneity in studied populations and coinvolved HCPs, the dissimilarity of included studies (Table 2 and, for background data, Appendix I, available in online article).

Patient-centered follow-up is the most deployed intervention ( $\mathrm{n}=19)$, followed by HCP-centered follow-up ( $\mathrm{n}=14$; Appendix J, available in online article). Other frequently used 


\begin{tabular}{|c|c|c|c|c|c|}
\hline $\begin{array}{l}\text { Intervention } \\
\text { Number }\end{array}$ & $\begin{array}{l}\text { Intervention } \\
\text { Category }\end{array}$ & Clarification & Target & Nature & Stage \\
\hline 1 & $\begin{array}{l}\text { Admission } \\
\text { reconciliation }\end{array}$ & $\begin{array}{l}\text { - All activities that led to assembling an accurate medication list, including a check for } \\
\text { appropriateness of prescribing and documentation of changes. }\end{array}$ & $\mathrm{HCP}$ & A & $\mathrm{OA}$ \\
\hline 2 & $\begin{array}{l}\text { Patient counseling } \\
\text { on admission }\end{array}$ & $\begin{array}{l}\text { - Actively incorporating the patient as a source (or recipient) of information. } \\
\text { - Patient counseling was not restricted to a certain stage, therefore, this intervention was } \\
\text { split to allow assignment to all stages (intervention numbers } 1,5 \text {, and } 7 \text { ). }\end{array}$ & $\mathrm{Pt}$ & $\mathrm{P}$ & $\mathrm{OA}$ \\
\hline 3 & $\begin{array}{l}\text { Pharmacist is part } \\
\text { of medical team }\end{array}$ & $\begin{array}{l}\text { - Pharmacist was an active member of the medical team, e.g., by participating in ward } \\
\text { rounds. }\end{array}$ & $\mathrm{HCP}$ & $\mathrm{P}$ & $\mathrm{DA}$ \\
\hline 4 & $\begin{array}{l}\text { Medication } \\
\text { review }\end{array}$ & $\begin{array}{l}\text { - According to Hatah et al., }{ }^{64} \text { medication review can be classified into } 4 \text { levels of compre- } \\
\text { hensiveness: (1) prescription review, (2) adherence support review (with patient present), } \\
\text { (3) clinical review, and (4) clinical review with prescribing. The latter } 2 \text { are conducted in } \\
\text { close collaboration with physicians. } \\
\text { - All levels were clustered in the PIM, but to prevent overclassification of lower levels, the } \\
\text { accompanying number is indicated in the results. }\end{array}$ & $\mathrm{HCP}$ & $\mathrm{P}$ & $\mathrm{DA}$ \\
\hline 5 & $\begin{array}{l}\text { Patient counseling } \\
\text { during admission }\end{array}$ & - See intervention 2. & $\mathrm{Pt}$ & $\mathrm{P}$ & DA \\
\hline 6 & $\begin{array}{l}\text { Discharge } \\
\text { reconciliation }\end{array}$ & - See intervention 1. & $\mathrm{HCP}$ & A & $\mathrm{AD}$ \\
\hline 7 & $\begin{array}{l}\text { Patient counseling } \\
\text { at discharge }\end{array}$ & - See intervention 2. & $\mathrm{Pt}$ & $\mathrm{P}$ & $\mathrm{AD}$ \\
\hline 8 & $\begin{array}{l}\text { Supplying patient } \\
\text { with discharge } \\
\text { letter }\end{array}$ & $\begin{array}{l}\text { - Providing the patient with a copy of the discharge letter to facilitate medication manage- } \\
\text { ment postdischarge. }\end{array}$ & $\mathrm{Pt}$ & A & $\mathrm{AD}$ \\
\hline 9 & $\begin{array}{l}\text { Transmission to } \\
\text { next HCP }\end{array}$ & - Transmission of an updated and verified medication list to next health care provider. & HCP & A & $\mathrm{AD}$ \\
\hline 10 & $\begin{array}{l}\text { Patient-centered } \\
\quad \text { follow-up }\end{array}$ & $\begin{array}{l}\text { - Postdischarge follow-up classification was based on intervention target. } \\
\text { - The subdivision in house calls }(\mathrm{H}) \text {, clinic visits }(\mathrm{C}) \text {, and telephone calls }(\mathrm{T}) \text { is indicated } \\
\text { in the results. } \\
\text { - Patient-centered follow-up comprises adherence counseling, for example. }\end{array}$ & $\mathrm{Pt}$ & $\mathrm{P}$ & PD \\
\hline 11 & $\begin{array}{l}\text { HCP-centered } \\
\text { follow-up }\end{array}$ & $\begin{array}{l}\text { - See intervention } 10 . \\
\text { - HCP-centered follow-up consists of reporting drug-related problems to general practitio- } \\
\text { ner, for example. }\end{array}$ & HCP & $\mathrm{P}$ & $\mathrm{PD}$ \\
\hline 12 & $\begin{array}{l}\text { Extra } \\
\text { postdischarge } \\
\text { follow-up }\end{array}$ & - Additional postdischarge follow-up to review progress and/or reinforce initial advice. & $\mathrm{Pt}$ & $\mathrm{P}$ & $\mathrm{PD}$ \\
\hline $13^{\mathrm{a}}$ & $\begin{array}{c}\text { Tailored } \\
\text { interventions }\end{array}$ & $\begin{array}{l}\text { - Interventions were tailored to individual patient's needs (e.g., cognition or low-literacy } \\
\text { skills). }\end{array}$ & $\mathrm{Pt}$ & $\mathrm{P}$ & SI \\
\hline $14 \mathrm{a}$ & $\begin{array}{l}\text { Provision of } \\
\text { adherence aids }\end{array}$ & - Supplying a pill box or a daily reminder routine, for example. & $\mathrm{Pt}$ & A & SI \\
\hline $15^{\mathrm{a}}$ & $\begin{array}{l}\text { Dispensing or } \\
\text { logistics aids }\end{array}$ & - Disposing of out-of-use or out-of-date medication, for example. & $\mathrm{Pt}$ & A & SI \\
\hline
\end{tabular}

aInterventions 13-15 were not bound to a certain stage and were therefore scored as stage independent.

$A=$ administrative $A D=$ at discharge $D A=$ during admission; $H C P=$ health care provider $; O A=$ on admission; $P=$ professional; $P D=$ postdischarge; $P I M=$ pharmacist intervention model; $P t=$ patient; $S I=$ stage independent .

interventions were medication review during admission $(n=13)$, patient counseling at discharge $(n=13)$, and admission reconciliation ( $\mathrm{n}=11$; Appendix J). Most studies measured readmissions $(n=24)$, followed by mortality $(n=15)$, emergency department visits $(n=10)$, and ADEs ( $n=4$; Table 2). Only 2 studies reported a composite outcome measurement (composite readmission/emergency department visits and composite readmission/emergency department visits/ mortality, respectively) impeding analysis of the individual outcomes. ${ }^{12,42}$

\section{Results of Individual Studies: Effectiveness of Interventions}

Study effects are shown in Table 2. All 30 studies showed a consistent effect on the outcomes included in this review. One study reported a significant increase in hospital readmissions at 6 months postdischarge. ${ }^{39}$ The average number of different pharmacist intervention components deployed in effective versus ineffective studies was approximately the same, 4.3 versus 5.1, respectively, as well as average patient-aimed (2.4 vs. 2.9), HCP-aimed (1.9 vs. 2.2), professional (2.9 vs. 3.0), and administrative intervention components (1.3 vs. 2.1). Finally, 


\section{FIGURE 1 Summary of Evidence} Search and Selection

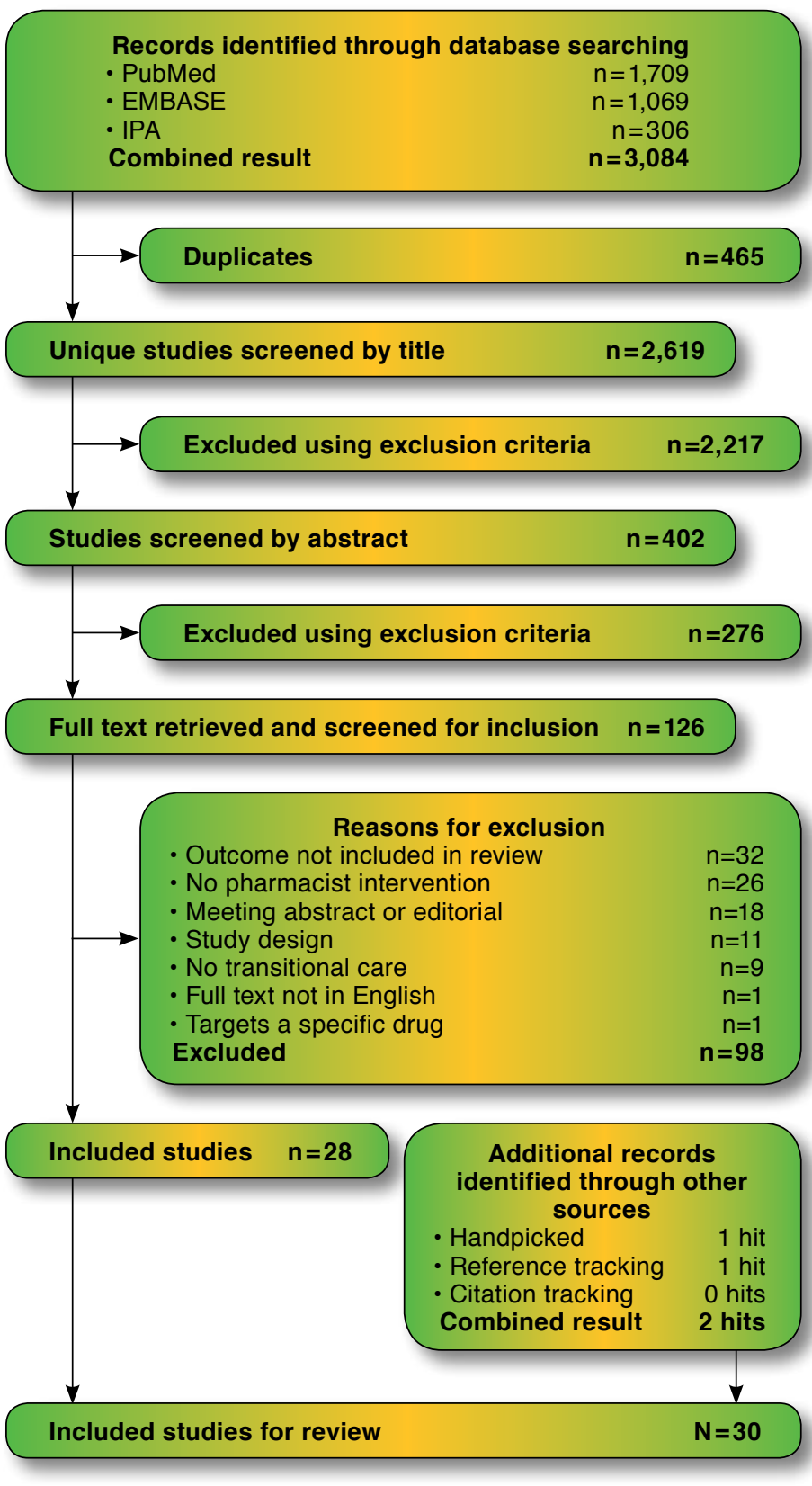

IPA = International Pharmaceutical Abstracts.

average-covered stages (1.9 vs. 2.1) and stage-independent interventions (0.6 vs. 0.6) were equally distributed, resulting in seemingly corresponding study characteristics regarding the deployed pharmacist interventions. All data were nonnormally distributed, and none of these differences were statistically significant $(P>0.05)$.

\section{Best Evidence Synthesis}

The cutoff point of $\geq 5$ bias-free domains yielded 15 studies, 9 effective and 6 ineffective, for the best evidence synthesis (Table 3). ${ }^{10,13,39-41,43,45,48,50-55,59}$ Levels of evidence were attributed to individual intervention components on improving the selected clinical outcomes.

Five studies, 3 effective and 2 ineffective, investigated the effect of isolated postdischarge intervention components indicating conflicting evidence. ${ }^{39-41,54,55}$ The studies' characteristics varied, since all 3 effective studies incorporated active nurse involvement either during the follow-up intervention or at hospital discharge. Furthermore, effective studies implemented individual patient tailoring, whereas ineffective studies had 1 or more additional follow-up contact moments.

Ten studies covered 1 or more hospital stages or combined in-hospital with postdischarge intervention components. By analyzing the individual components of these multifaceted interventions, different best evidence synthesis levels could be assigned. There is strong evidence for active pharmacist involvement during admission by performing a level 3 medication review. This level also requires active physician involvement (Table 3). Five effective studies incorporated this intervention component, compared with none of the ineffective studies. ${ }^{10,50-53}$ In 2 out of these 5 effective studies, the pharmacist was also part of the multidisciplinary medical team. ${ }^{10,50}$ All other intervention components revealed conflicting evidence. Five studies ( 3 effective and 2 ineffective) incorporated medication reconciliation on admission. ${ }^{10,13,52,53,59}$ Two of the effective studies combined reconciliation with patient counseling on admission. ${ }^{10,53}$ Five studies ( 3 effective and 2 ineffective) covered 3 or more stages from hospital admission to postdischarge follow-up..$^{10,13,51,53,59}$ Finally, 7 studies (3 effective and 4 ineffective) combined a postdischarge intervention with 1 or more in-hospital intervention. ${ }^{10,13,43,45,48,51,59}$ The postdischarge intervention components varied mainly in thoroughness.

\section{Discussion}

All included studies varied regarding the type and moment of intervention, studied population, involvement of other health care providers, and selected outcomes. This variability is reflected in the clinical outcomes of these studies. Although this heterogeneity resulted in inconclusiveness of our predefined clustering to elucidate the most effective intervention components, the heterogeneity also enabled a best evidence synthesis. This synthesis suggests that for an isolated postdischarge program, pharmacists are most likely to contribute to improved patient outcomes by closely collaborating with nurses. Moreover, in multifaceted programs, pharmacists have additional value by performing a clinical medication review in addition to patient-involved medication reconciliation followed up by a thorough postdischarge intervention. Finally, the best evidence synthesis suggests that these pharmacist 
TABLE 2 Characteristics of Studies Included in Systematic Review

\begin{tabular}{|c|c|c|c|c|}
\hline Author/Date & $\begin{array}{l}\text { Setting (Country/Ward/Hospital Type/Sample } \\
\text { Size + High-Risk Selection, If Applicable) }\end{array}$ & Outcome $^{a}$ & Time & $\begin{array}{c}\text { Effect } \\
(\%)\end{array}$ \\
\hline \multirow{2}{*}{$\begin{array}{l}\text { Al-Rashed et al., } \\
2002^{58}\end{array}$} & \multirow[t]{2}{*}{ GBR/elderly ward/general hospital/83 patients } & Readmissions & 15-22 days & $\downarrow 62$ \\
\hline & & Readmissions & 3 months & $\downarrow 80$ \\
\hline \multirow[t]{2}{*}{ Barker et al., $2012^{37}$} & \multirow{2}{*}{$\begin{array}{l}\text { AUS/all wards/teaching hospital/114 CHF } \\
\text { patients }\end{array}$} & Mortality & 6 months & $\uparrow 41$ \\
\hline & & Readmissions & 6 months & $\uparrow 18$ \\
\hline Bolas et al., 200438 & $\begin{array}{l}\text { GBR/medical admission ward/general } \\
\text { hospital/162 acutely admitted patients }\end{array}$ & Readmissions (emergency) & 3 months & NR \\
\hline \multirow[t]{2}{*}{ Dudas et al., $2001^{36}$} & \multirow{2}{*}{$\begin{array}{l}\text { USA/general medicine ward/academic } \\
\text { hospital/145 patients }\end{array}$} & Readmissions & 30 days & $\downarrow 40$ \\
\hline & & ED visits & 30 days & $\downarrow 58$ \\
\hline \multirow{3}{*}{$\begin{array}{l}\text { Englander et al., } \\
20146\end{array}$} & \multirow{3}{*}{$\begin{array}{l}\text { USA/general medicine \& cardiology wards/ } \\
\text { academic hospital/382 patients }\end{array}$} & Mortality & 30 days & $\downarrow 100$ \\
\hline & & Readmissions & 30 days & $\downarrow 11$ \\
\hline & & ED visits & 30 days & $\downarrow 24$ \\
\hline \multirow[t]{12}{*}{ Farris et al, 201459} & \multirow{12}{*}{$\begin{array}{l}\text { USA/a general medicine, family medicine, } \\
\text { cardiology, or orthopedics ward/academic } \\
\text { hospital/936 patients with a predefined } \\
\text { chronic condition }\end{array}$} & $\begin{array}{l}\text { Readmissions (inpatient \& outpatient } \\
\text { intervention) }\end{array}$ & 30 days & $\uparrow 14$ \\
\hline & & Readmissions (inpatient intervention) & 30 days & $\downarrow 8$ \\
\hline & & $\begin{array}{l}\text { Readmissions (inpatient } \& \text { outpatient } \\
\text { intervention) }\end{array}$ & 90 days & $\uparrow 9$ \\
\hline & & Readmissions (inpatient intervention) & 90 days & $\uparrow 9$ \\
\hline & & ED visits (inpatient \& outpatient intervention) & 30 days & $\downarrow 24$ \\
\hline & & ED visits (inpatient intervention) & 30 days & $\downarrow 7$ \\
\hline & & ED visits (inpatient \& outpatient intervention) & 90 days & $\perp 7$ \\
\hline & & ED visits (inpatient intervention) & 90 days & $\downarrow 13$ \\
\hline & & ADEs (inpatient \& outpatient intervention) & on admission & $\downarrow 15$ \\
\hline & & ADEs (inpatient \& outpatient intervention) & at discharge & $\uparrow 36$ \\
\hline & & ADEs (inpatient intervention) & 90 days & 18 \\
\hline & & ADEs (inpatient \& outpatient intervention) & 90 days & $\perp 5$ \\
\hline \multirow{5}{*}{ Gillespie et al., 200910} & \multirow{5}{*}{$\begin{array}{l}\text { SWE/internal medicine ward/academic } \\
\text { hospital/368 acutely admitted patients }\end{array}$} & Mortality & 12 months & $\uparrow 3$ \\
\hline & & Readmissions & 12 months & $\$ 3$ \\
\hline & & Readmissions (drug related) & 12 months & $\downarrow 80$ \\
\hline & & ED visits & 12 months & $\downarrow 47$ \\
\hline & & Composite RE & 12 months & $\downarrow 16$ \\
\hline $\begin{array}{l}\text { Gwadry-Sridhar et al., } \\
2005^{12}\end{array}$ & $\begin{array}{l}\text { CAN/acute medical \& surgical wards/teaching } \\
\text { hospital/134 CHF patients }\end{array}$ & Composite REM & 12 months & $\downarrow 10$ \\
\hline \multirow[t]{6}{*}{ Hawes et al., 201460} & \multirow{6}{*}{$\begin{array}{l}\text { USA/family medicine ward/academic hospital/61 } \\
\text { patients with a predefined chronic condition }\end{array}$} & Readmissions (intention to treat) & 30 days & $\downarrow 100$ \\
\hline & & Readmissions (per protocol) & 30 days & $\downarrow 100$ \\
\hline & & ED visits (intention to treat) & 30 days & $\downarrow 100$ \\
\hline & & ED visits (per protocol) & 30 days & $\downarrow 100$ \\
\hline & & Composite RE (intention to treat) & 30 days & $\downarrow 100$ \\
\hline & & Composite RE (per protocol) & 30 days & $\downarrow 100$ \\
\hline \multirow[t]{2}{*}{ Holland et al., $2005^{39}$} & \multirow{2}{*}{$\begin{array}{l}\text { GBR/all wards/several general hospitals/829 } \\
\text { acutely admitted patients }\end{array}$} & Mortality & 6 months & $\downarrow 22$ \\
\hline & & Readmissions (emergency) & 6 months & $\uparrow 31$ \\
\hline \multirow[t]{2}{*}{ Holland et al., $2007^{40}$} & \multirow{2}{*}{$\begin{array}{l}\text { GBR/all wards/3 general hospitals/291 acutely } \\
\text { admitted CHF patients }\end{array}$} & Mortality & 6 months & $\uparrow 25$ \\
\hline & & Readmissions & 6 months & $\uparrow 20$ \\
\hline \multirow[t]{3}{*}{ Jack et al., $2009^{41}$} & \multirow[t]{3}{*}{ USA/all wards/academic hospital/738 patients } & Readmissions & 30 days & $\downarrow 28$ \\
\hline & & ED visits & 30 days & $\downarrow 32$ \\
\hline & & Composite RE & 30 days & $\$ 30$ \\
\hline \multirow[t]{2}{*}{ Koehler et al., $2009^{42}$} & USA/hospital-medicine ward/academic hospital/ & Composite RE & 3 months & $\downarrow 74$ \\
\hline & 41 patients more than 3 chronic conditions & Composite RE & 6 months & $\uparrow 300$ \\
\hline Kripalani et al., $2012^{13}$ & USA/cardiology ward/2 academic hospitals/851 & ADEs (preventable) & 30 days & $\uparrow 8$ \\
\hline & CHF patients & ADEs (potential) & 30 days & $\$ 20$ \\
\hline Lipton and Bird, & USA/all wards (except psychiatry)/general & Readmissions (emergency) & 1 months & NR \\
\hline 199443 & hospital/706 patients & Readmissions (emergency) & 3 months & NR \\
\hline & & Readmissions (emergency) & 6 months & NR \\
\hline Lisby et al., $2010^{44}$ & DNK/internal medicine ward/general hospital/99 & Mortality & 3 months & $\uparrow 60$ \\
\hline & acutely admitted patients & Readmissions & 3 months & $\downarrow 20$ \\
\hline & & ED visits & 3 months & $\leftrightarrow 0$ \\
\hline
\end{tabular}


TABLE 2 Characteristics of Studies Included in Systematic Review (continued)

\begin{tabular}{|c|c|c|c|c|}
\hline Author/Date & $\begin{array}{c}\text { Setting (Country/Ward/Hospital Type/Sample } \\
\text { Size + High-Risk Selection, If Applicable) }\end{array}$ & Outcome $^{a}$ & Time & $\begin{array}{c}\text { Effect } \\
(\%)\end{array}$ \\
\hline \multirow{6}{*}{$\begin{array}{l}\text { López Cabezas et al., } \\
2006^{45}\end{array}$} & \multirow{6}{*}{$\begin{array}{l}\text { ESP/cardiology \& internal medicine wards/2 } \\
\text { general hospitals/134 CHF patients }\end{array}$} & Mortality & 2 months & $\downarrow 83$ \\
\hline & & Mortality & 6 months & $\downarrow 50$ \\
\hline & & Mortality & 12 months & $\downarrow 53$ \\
\hline & & Readmissions & 2 months & $\downarrow 68$ \\
\hline & & Readmissions & 6 months & $\downarrow 57$ \\
\hline & & Readmissions & 12 months & $\downarrow 50$ \\
\hline \multirow{2}{*}{$\begin{array}{l}\text { Makowsky et al., } \\
2009^{46}\end{array}$} & \multirow{2}{*}{$\begin{array}{l}\text { CAN/acute internal medicine } \& \text { family medicine } \\
\text { wards/3 teaching hospitals/451 patients with a } \\
\text { predefined chronic condition }\end{array}$} & Readmissions & 3 months & $\downarrow 20$ \\
\hline & & Readmissions & 6 months & $\downarrow 10$ \\
\hline \multirow{2}{*}{$\begin{array}{l}\text { Naunton et al., } \\
2003^{47}\end{array}$} & \multirow{2}{*}{$\begin{array}{l}\text { AUS/all medical wards/teaching hospital/121 } \\
\text { patients with more than } 2 \text { medication-requiring } \\
\text { chronic conditions }\end{array}$} & Mortality & 3 months & $\downarrow 38$ \\
\hline & & Readmissions & 3 months & $\downarrow 38$ \\
\hline \multirow{4}{*}{$\begin{array}{l}\text { Nazareth et al., } \\
2001^{48}\end{array}$} & \multirow{4}{*}{$\begin{array}{l}\text { GBR/geriatric medicine ward/4 general } \\
\text { hospitals/347 patients }\end{array}$} & Mortality & 3 months & $\uparrow 116$ \\
\hline & & Mortality & 6 months & $\uparrow 28$ \\
\hline & & Readmissions & 3 months & $\leftrightarrow 0$ \\
\hline & & Readmissions & 6 months & $\downarrow 2$ \\
\hline \multirow[t]{2}{*}{ Rainville, 199949} & \multirow[t]{2}{*}{ USA/all wards/tertiary hospital/34 CHF patients } & Readmissions (HF related) & 12 months & $\downarrow 60$ \\
\hline & & Composite REM (HF related) & 12 months & $\downarrow 64$ \\
\hline \multirow{6}{*}{$\begin{array}{l}\text { Schmader et al., } \\
200450\end{array}$} & \multirow{6}{*}{$\begin{array}{l}\text { USA/all wards/1l Veterans Affairs hospitals/834 } \\
\text { patients meeting frailty criteria }\end{array}$} & ADEs (all, inpatient intervention) & at discharge & $\uparrow 222$ \\
\hline & & ADEs (serious, inpatient intervention) & at discharge & $\uparrow 80$ \\
\hline & & ADEs (all, inpatient intervention) & 12 months & $\downarrow 18$ \\
\hline & & ADEs (serious, inpatient intervention) & 12 months & $\downarrow 19$ \\
\hline & & ADEs (all, outpatient intervention) & 12 months & $\downarrow 1$ \\
\hline & & ADEs (serious, outpatient intervention) & 12 months & $\downarrow 37$ \\
\hline \multirow{4}{*}{$\begin{array}{l}\text { Schnipper et al., } \\
2006^{51}\end{array}$} & \multirow{4}{*}{$\begin{array}{l}\text { USA/general medicine ward/teaching hospital/ } \\
152 \text { patients }\end{array}$} & Composite RE & 30 days & $\leftrightarrow 0$ \\
\hline & & Composite RE (drug related) & 30 days & $\downarrow 50$ \\
\hline & & Composite RE (preventable drug related) & 30 days & $\downarrow 88$ \\
\hline & & ADEs (preventable) & 30 days & $\downarrow 88$ \\
\hline \multirow{2}{*}{$\begin{array}{l}\text { Schnipper et al., } \\
2009^{52}\end{array}$} & \multirow{2}{*}{$\begin{array}{l}\text { USA/general medicine ward/2 general hospitals/ } \\
322 \text { patients }\end{array}$} & Composite RE & 30 days & $\downarrow 17$ \\
\hline & & ADEs (potential) & at discharge & $\downarrow 26$ \\
\hline \multirow[t]{2}{*}{ Scullin et al., $2007^{53}$} & \multirow{2}{*}{$\begin{array}{l}\text { GBR/2 medical wards/3 general hospitals/762 } \\
\text { patients }\end{array}$} & Mortality & 12 months & $\downarrow 8$ \\
\hline & & Readmissions & 12 months & $\downarrow 17$ \\
\hline \multirow{3}{*}{$\begin{array}{l}\text { Spinewine et al., } \\
2007^{11}\end{array}$} & \multirow{3}{*}{$\begin{array}{l}\text { BEL/geriatric medicine ward/academic hospital/ } \\
186 \text { patients }\end{array}$} & Mortality & 12 months & $\downarrow 25$ \\
\hline & & Readmissions & 12 months & $\$ 3$ \\
\hline & & ED visits & 12 months & $\downarrow 34$ \\
\hline \multirow{4}{*}{$\begin{array}{l}\text { Stewart et al., } \\
1998^{55}\end{array}$} & \multirow[t]{4}{*}{ AUS/all wards/tertiary hospital/762 patients } & Mortality & 6 months & $\downarrow 59$ \\
\hline & & Readmissions & 6 months & $\downarrow 22$ \\
\hline & & ED visits & 6 months & $\downarrow 25$ \\
\hline & & Composite RM & 6 months & $\downarrow 29$ \\
\hline \multirow{4}{*}{$\begin{array}{l}\text { Stewart et al., } \\
1998^{54}\end{array}$} & AUS/all wards/tertiary hospital/97 CHF patients & Mortality & 6 months & $\downarrow 50$ \\
\hline & & Readmissions & 6 months & $\downarrow 23$ \\
\hline & & ED visits & 6 months & $\downarrow 45$ \\
\hline & & Composite RM & 6 months & $\downarrow 43$ \\
\hline Stowasser et al., & AUS/all acute wards $\&$ orthopedics ward/2 & Mortality & 30 days & $\downarrow 25$ \\
\hline $2002^{56}$ & general hospitals/240 patients & Readmissions & 30 days & $\downarrow 74$ \\
\hline & & Readmissions (emergency) & 30 days & $\downarrow 38$ \\
\hline Triller et al., $2007^{57}$ & USA/all wards/2 general hospitals/154 CHF & Mortality & 6 months & $\uparrow 21$ \\
\hline & patients & Readmissions & 6 months & $\downarrow 9$ \\
\hline
\end{tabular}

Note: Italicized entries indicate $P<0.05$.

${ }^{a}$ Outcomes reported as all-cause if not stated otherwise.

$A D E s=a d v e r s e$ drug events; $A U S=$ Australia; $B E L=$ Belgium; $C A N=$ Canada $; C H F=$ congestive heart failure; $D N K=$ Denmark; $E D=$ emergency department; ESP = Spain; $G B R=$ United Kingdom; $H C P=$ health care provider; $H F=$ heart failure; $R E=$ readmissions and $E D$ visits; $R E M=$ readmissions, $E D$ visits, and mortality; NR=not reported; RM= readmissions and mortality; SWE = Sweden; USA=United States of America. 
TABLE 3 Best Evidence Synthesis: Effective (Upper-Part) Versus Ineffective (Lower-Part) Studies, Sorted by Bias-Free Domains

\begin{tabular}{|c|c|c|c|c|c|c|c|c|c|c|c|c|c|c|c|c|c|}
\hline \multicolumn{3}{|r|}{ Study Characteristics } & \multicolumn{15}{|c|}{ Pharmacist Intervention } \\
\hline Author/Date & $\begin{array}{l}\text { Bias-Free } \\
\text { Domains }\end{array}$ & Coinvolved Health Care Provider & 1 & 2 & 3 & $4^{a}$ & 5 & 6 & 7 & 8 & 9 & $10^{\mathrm{b}}$ & 11 & 12 & 13 & 14 & 15 \\
\hline $\begin{array}{l}\text { Jack et al., } \\
2009^{41}\end{array}$ & 6 & $\begin{array}{l}\text { N: coordination, counseling } \\
\text { PCP: act on drug-related recommendations } \\
\text { (PD) }\end{array}$ & & & & & & & & & & $\mathbf{T}$ & $\bullet$ & & & & \\
\hline $\begin{array}{l}\text { Stewart et al., } \\
1998^{54}\end{array}$ & 6 & $\mathrm{~N}$ : counseling, risk assessment, and referral & & & & & & & & & & $\mathbf{H}$ & $\bullet$ & & $\bullet$ & $\bullet$ & \\
\hline $\begin{array}{l}\text { Stewart et al., } \\
1998^{55}\end{array}$ & 6 & $\mathrm{~N}$ : counseling, risk assessment, and referral & & & & & & & & & & $\mathbf{H}$ & $\bullet$ & & • & $\bullet$ & \\
\hline $\begin{array}{l}\text { Gillespie et al., } \\
200910\end{array}$ & 5 & $\begin{array}{l}\text { S: act on drug-related recommendations } \\
\text { (DA) }\end{array}$ & $\bullet$ & $\bullet$ & $\bullet$ & 3 & $\bullet$ & & $\bullet$ & $\bullet$ & $\bullet$ & $\mathbf{T}$ & & & - & & \\
\hline $\begin{array}{l}\text { López Cabezas } \\
\text { et al., } 2006^{45}\end{array}$ & 5 & None & & & & & & & $\bullet$ & & & $\mathbf{T}$ & & $\bullet$ & & & \\
\hline $\begin{array}{l}\text { Schmader et al., } \\
200450\end{array}$ & 5 & $\begin{array}{l}\text { S: act on drug-related recommendations } \\
\text { (DA) }\end{array}$ & & & $\bullet$ & 3 & & & & & & & & & & & \\
\hline $\begin{array}{l}\text { Schnipper et al., } \\
2006^{51}\end{array}$ & 5 & $\begin{array}{l}\text { S: act on drug-related recommendations } \\
\text { (DA) } \\
\text { PCP: act on drug-related recommendations } \\
\text { (PD) }\end{array}$ & & & & 3 & & $\bullet$ & 0 & & & $\mathbf{T}$ & - & & & & \\
\hline $\begin{array}{l}\text { Schnipper et al., } \\
2009^{52}\end{array}$ & 5 & $\begin{array}{l}\text { S: reconciliation, act on drug-related } \\
\text { recommendations (DA) } \\
\text { N: counseling }\end{array}$ & - & & & 3 & & & & & & & & & & & \\
\hline $\begin{array}{l}\text { Scullin et al., } \\
2007^{53}\end{array}$ & 5 & $\begin{array}{l}\text { S: act on drug-related recommendations } \\
\text { (DA) }\end{array}$ & $\bullet$ & 0 & & 3 & 0 & 0 & $\bullet$ & 0 & ○ & & & & 0 & & 0 \\
\hline $\begin{array}{l}\text { Farris et al., } \\
201459\end{array}$ & 6 & $\begin{array}{l}\text { PCP: act on drug-related recommendations } \\
\text { (PD) }\end{array}$ & $\bullet$ & & & & 0 & $\bullet$ & $\bullet$ & $\bullet$ & $\bullet$ & $\mathbf{T}$ & 0 & $\bullet$ & & $\bullet$ & \\
\hline $\begin{array}{l}\text { Holland et al., } \\
2005^{39}\end{array}$ & 6 & $\begin{array}{l}\text { PCP: act on drug-related recommendations } \\
\text { (PD) }\end{array}$ & & & & & & & & & & $\mathbf{H}$ & • & $\bullet$ & & & 0 \\
\hline $\begin{array}{l}\text { Holland et al., } \\
200740\end{array}$ & 6 & $\begin{array}{l}\text { PCP: act on drug-related recommendations } \\
\text { (PD) }\end{array}$ & & & & & & & & & & H & • & $\bullet$ & & & • \\
\hline $\begin{array}{l}\text { Kripalani et al., } \\
2012^{13}\end{array}$ & 6 & $\begin{array}{l}\text { SW: coordination } \\
\text { PCP: act on drug-related recommendations } \\
\text { (PD) }\end{array}$ & $\bullet$ & & & 2 & 0 & & 0 & $\bullet$ & & $\mathbf{T}$ & • & & $\bullet$ & 0 & \\
\hline $\begin{array}{l}\text { Nazareth et al., } \\
2001^{48}\end{array}$ & 6 & $\begin{array}{l}\text { PCP: act on drug-related recommendations } \\
\text { (PD) }\end{array}$ & & & & & & $\bullet$ & - & 0 & - & $\mathbf{H}$ & - & & 0 & & \\
\hline $\begin{array}{l}\text { Lipton and Bird, } \\
199443\end{array}$ & 5 & $\begin{array}{l}\text { PCP: act on drug-related recommendations } \\
\text { (PD) }\end{array}$ & & & & & & 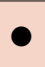 & $\bullet$ & & & $\mathbf{T}$ & - &  & & & \\
\hline
\end{tabular}

${ }^{a}$ Medication review levels: $2=$ adherence support review (with patient present), $3=$ clinical review.

${ }^{b} \mathrm{H}=$ home visit; $\mathrm{T}=$ telephone call.

$D A=$ during admission; $N=$ nurse; $P C P=$ primary care physician; $P D=$ postdischarge; $S=$ specialist $S W=$ social worker.

interventions are especially effective when performed in close collaboration with physicians.

The conflicting evidence regarding studies investigating an isolated postdischarge intervention possibly originates from the variances in study conditions. ${ }^{39-41,54,55}$ As mentioned earlier, all 3 effective studies involved a pharmacist's follow-up in close collaboration with a nurse. In 2 studies, this involvement was realized by performing a home-based follow-up as a pharmacist-nurse team. ${ }^{54,55}$ In those cases, the nurse focused on detecting any clinical deterioration, whereas the pharmacist focused mainly on adherence counseling and adequate monitoring by caregivers. If necessary, both HCPs deployed a subsequent referral to either the general practitioner or community pharmacist, respectively. The third study brought nurses into action to coordinate the discharge plan with the hospital team and educate and prepare patients for discharge. ${ }^{41}$ These results indicate the need for a multidisciplinary intervention, which is in agreement with earlier findings. ${ }^{19}$ Next, tailoring the intervention, for instance by assessing patient knowledge of the prescribed medications and compliance, was utilized in all effective studies (in Jack et al., 2009, ${ }^{41}$ nurses used tailored intervention during hospital discharge). This practice is in contrast with the ineffective studies, which had the more general approach of offering medication boxes to every patient involved, for example. ${ }^{39,40,61}$ The need for tailoring intervention to patient needs is further illustrated by the ineffective studies, 
which deployed additional follow-up visits to reinforce original advice. Although in itself this might be a valuable intervention component, it should possibly be tailored to specific patient needs or population to optimize its efficacy. Finally, effective studies deployed a pharmacist from the involved hospital, whereas ineffective studies deployed an external research pharmacist who was neither familiar with the patients' home situations nor the previous hospital stays. Hence, as hospital discharge is well known to be confusing and distressing, ${ }^{62,63}$ this might increase the risk of discontinuity of care.

Regarding the multifaceted programs, several implications for daily practice can be extracted. Although individual effects of multifaceted interventions are difficult to determine, the best evidence synthesis imposed strong evidence for the effectiveness of medication review during hospital admission. Six multifaceted intervention programs incorporated this intervention component, but the level of assessing medication appropriateness varies between studies. ${ }^{10,13,50-53,64}$ The 5 effective studies performed a rigorous clinical medication review (level 3) as compared with 1 ineffective study that performed an adherence support review (level 2). A level 3 review aimed at optimization of pharmacotherapy with access to clinical notes enables pharmacists to address the patients' use of medications in the context of their clinical conditions. Furthermore, a level 3 medication review during hospital admission requires a close collaboration with the responsible physician. Since only the effective studies incorporated this rigorous review, the beneficial effect might also be attributed to the multidisciplinary collaboration between pharmacists and physicians.

Several multifaceted intervention programs deployed medication reconciliation on admission but differed in setting by active patient involvement. ${ }^{10,13,52,53,59}$ A recent report by the Agency for Healthcare Research and Quality (AHRQ) evaluated the role of pharmacists during care transition programs. ${ }^{65}$ This report recommends medication reconciliation during care transition to minimize risks. However, to obtain a best possible medication history, medication reconciliation needs to be supplemented with a structured patient interview. ${ }^{16}$ All 3 effective studies included this intervention, either by the pharmacist or by the attending physician, in contrast to the 2 ineffective studies that did not include the interview. ${ }^{13,59}$ This indicates that a structured patient interview needs to be part of admission reconciliation, but it does not necessarily need to be conducted by a pharmacist.

Kwan et al. (2013) suggested that performing medication reconciliation alone is not sufficient to reduce postdischarge clinical outcomes (e.g., hospital readmission), since it needs to be combined with other interventions aimed at care transition improvement. ${ }^{16}$ By incorporating an extensive combination of pharmacist interventions in hospital and primary health care settings, continuity of care can be secured. ${ }^{24}$ In this review, 7 studies combined a postdischarge intervention with 1 or more in-hospital interventions. ${ }^{10,13,43,45,48,51,59}$ Three studies covered all stages from hospital admission to postdischarge followup. ${ }^{10,13,59}$ Analysis of the in-hospital intervention components of Gillespie et al. (2009)10 —one of the effective studies-revealed that pharmacists participated in ward rounds and as members of the medical team, in contrast to the ineffective studies..$^{13,59}$ During those ward rounds, pharmacists discussed the identified drug-related problems with the responsible physicians, which possibly improved implementation of the pharmacists' recommendations. Moreover, at hospital discharge, Gillespie et al. provided the patients' general practitioner with an extensive pharmacist discharge letter containing all in-hospital changes (with rationale), monitoring needs, expected therapeutic goals, and outstanding drug-related problems (with suggested actions). ${ }^{10}$ Farris et al. (2014) deployed a similar intervention, but the ineffectiveness could be attributed to the use of a research pharmacist (as mentioned earlier) or lack of contrast between intervention and control groups..$^{59}$ So, to be successful in reducing clinical outcomes such as hospital readmission, a more extensive pharmacist presence during all stages might be beneficial. However, it is crucial that the pharmacist acts in close collaboration with either the hospital-based team or the primary care provider.

In-depth analysis of the design of the postdischarge interventions in the 7 multifaceted programs that combined a postdischarge intervention with 1 or more in-hospital interventions showed great variance. ${ }^{10,13,43,45,48,51,59}$ The effective studies-Gillespie et al. ${ }^{10}$ and López Cabezas et al. (2006) ${ }^{45}$ - used a follow-up telephone call to reinforced in-hospital provided interventions, and Schnipper et al. (2006) ${ }^{51}$ combined telephone reinforcement with active feedback to primary care providers. Regarding the ineffective studies, Nazareth et al. $(2001)^{48}$ supplied the community pharmacists with only the patients' discharge medication regimens and focused on patient compliance and knowledge during the pharmacist house call. Although the intervention was fairly thorough, the community pharmacists were not supplied with the patients' previous clinical histories. Another ineffective study, Kripalani et al. (2012) ${ }^{13}$ incorporated a pharmacist follow-up telephone call "as needed," risking the possibility of missing relevant interventions, and the results of Farris et al ${ }^{59}$ might be flawed by a less rigorous implementation of medication-related recommendations postdischarge. Finally, Lipton and Bird (1994) ${ }^{43}$ focused mainly on compliance by reducing regimen complexity during a telephone call. So, although not conclusive, evidence tends towards performing a comprehensive postdischarge follow-up based on previous in-hospital interventions by a pharmacist who is equipped with the patient's previous medical history. 


\section{Limitations}

This review has several strengths. First, the comprehensive search strategy utilized an automated database search of 3 pharmacy-relevant databases with manual reference tracking, which resulted in a complete overview of published studies in this field. Next, all articles were screened and extracted independently by 2 reviewers, ensuring that a solid selection of relevant studies and study characteristics were identified. Finally, because of a detailed data extraction process, it was possible to separate the various pharmacist intervention components.

First, an important limitation of this review is the risk of underreporting the deployed intervention components because of a possible lack of detailed descriptions in the original articles. Since only data from the original articles were extracted for the pharmacist intervention model, important components may have been missed. Second, although a comprehensive literature search was performed, publication bias is an important potential source of bias in systematic reviews. ${ }^{31}$ Therefore, unpublished research was not included in our analysis. Third, the selected clinical outcomes for this review were not always the primary outcomes of the included studies, which might result in an included trial being underpowered. Although most included studies were effective on surrogate endpoints (e.g., knowledge or adherence), by excluding these data, only clinically relevant outcomes were investigated implying strong evidence. Finally, we included only articles published in English and may therefore have missed some relevant literature.

\section{Conclusions}

Pharmacists can successfully perform interventions across different health care settings. ${ }^{5,66}$ Although there is a need for well-designed and well-reported RCTs, this systematic review indicates several pharmacist intervention components that could reduce the risks involved during care transitions. When performing an isolated postdischarge intervention, evidence tends towards collaborating with nurses and tailoring interventions to individual patient needs. In multifaceted intervention programs, performing medication reconciliation alone is possibly insufficient in reducing postdischarge clinical outcomes and should be combined with active patient counseling and a clinical medication review during admission. Furthermore, close collaboration between pharmacists and physicians during all stages of hospitalization is beneficial. Finally, it is important to secure continuity of care by integrating an outreaching hospital pharmacist or a community pharmacist in these multifaceted programs across the health care settings. Ultimately, the pharmacist involved in the intervention needs to be provided with the patient's clinical background and previous hospital experience.

\section{Authors}

HENDRIK T. ENSING, PharmD, is Researcher, Research Group Process Innovations in Pharmaceutical Care, Utrecht University of Applied Sciences; PhD Candidate, Department of Pharmacoepidemiology \& Clinical Pharmacology, Utrecht Institute for Pharmaceutical Sciences, Utrecht, the Netherlands; and Community Pharmacist, Zorggroep Almere, Outpatient Pharmacy "de Brug 24/7," Almere, the Netherlands. CLEMENTINE C. M. STUIJT, PharmD, is Clinical Pharmacist, ApoMed, Amsterdam, the Netherlands; BART J. F. VAN DEN BEMT, PharmD, PhD, is Pharmacist, Department of Pharmacy, Sint Maartenskliniek, Nijmegen, and Clinical Scientist, Radboud University Medical Center, Nijmegen, the Netherlands; AD A. VAN DOOREN, PharmD, PhD, is Professor, Research Group Process Innovations in Pharmaceutical Care, Utrecht University of Applied Sciences, Utrecht, the Netherlands; and FATMA KARAPINAR-ÇARKIT, PharmD, PhD, is Hospital Pharmacist/Epidemiologist, Department of Clinical Pharmacy, Sint Lucas Andreas Hospital, Amsterdam, the Netherlands. ELLEN S. KOSTER, PhD, is Assistant Professor/ Epidemiologist, and MARCEL L. BOUVY, PharmD, PhD, is Professor, Department of Pharmacoepidemiology \& Clinical Pharmacology, Utrecht Institute for Pharmaceutical Sciences, Utrecht, the Netherlands.

AUTHOR CORRESPONDENCE: Hendrik T. Ensing, PharmD, Research Group Process Innovations in Pharmaceutical Care, Utrecht University of Applied Sciences, Bolognalaan 101, P.O. Box 85182, 3508 AD Utrecht, the Netherlands. Tel.: +31 (0)6-480 796 16; Fax:+31 (0)36-54 54 377; E-mail: rensing@zorggroep-almere.nl.

\section{DISCLOSURES}

No funds were received for conducting this review. The authors attest that they have no financial interests, arrangements, or other conflicts of interest to disclose.

Bouvy had full access to all of the data in the study and takes responsibility for the integrity of the data and the accuracy of the data analysis. Study concept and design were contributed by Ensing, Stuijt, Koster, and Bouvy. Data were collected by Ensing and Stuijt and interpretated by Ensing, Stuijt, Karapinar-Çarkit, Koster, and Bouvy. The manuscript was written by Ensing, Stuijt, Koster, and Bouvy and revised by van den Bemt, van Dooren, Karapinar-Çarkit, and Koster.

\section{REFERENCES}

1. Borgsteede SD, Karapinar-Çarkit F, Hoffmann E, Zoer J, van den Bemt PM. Information needs about medication according to patients discharged from a general hospital. Patient Educ Couns. 2011;83(1):22-28.

2. Mueller SK, Sponsler KC, Kripalani S, Schnipper JL. Hospital-based medication reconciliation practices: a systematic review. Arch Intern Med. 2012;172(14):1057-69.

3. Witherington EM, Pirzada OM, Avery AJ. Communication gaps and readmissions to hospital for patients aged 75 years and older: observational study. Qual Saf Health Care. 2008;17(1):71-75.

4. Poston KM, Dumas BP, Edlund BJ. Outcomes of a quality improvement project implementing stroke discharge advocacy to reduce 30-day readmission rates. J Nurs Care Qual. 2014;29(3):237-44. 
5. White CM. Pharmacists need recognition as providers to enhance patient care. Ann Pharmacother. 2014;48(2):268-73.

6. Englander H, Michaels L, Chan B, Kansagara D. The care transitions innovation (C-TraIn) for socioeconomically disadvantaged adults: results of a cluster randomized controlled trial. J Gen Intern Med. 2014;29(11):1460-67.

7. Harrison JD, Auerbach AD, Quinn K, Kynoch E, Mourad M. Assessing the impact of nurse post-discharge telephone calls on 30-day hospital readmission rates. J Gen Intern Med. 2014;29(11):1519-25.

8. Institute of Medicine. Crossing the Quality Chasm: A New Health System for The 21st Century. Washington, DC: National Academies Press; 2001.

9. Kohn LT, Corrigan JM, Donaldson MS, eds. To Err Is Human: Building a Safer Health System. Washington, DC: National Academies Press; 2000.

10. Gillespie U, Alassaad A, Henrohn D, et al. A comprehensive pharmacist intervention to reduce morbidity in patients 80 years or older: a randomized controlled trial. Arch Intern Med. 2009;169(9):894-900.

11. Spinewine A, Swine C, Dhillon S, et al. Effect of a collaborative approach on the quality of prescribing for geriatric inpatients: a randomized, controlled trial. J Am Geriatr Soc. 2007;55(5):658-65.

12. Gwadry-Sridhar FH, Arnold JM, Zhang Y, Brown JE, Marchiori G, Guyatt G. Pilot study to determine the impact of a multidisciplinary educational intervention in patients hospitalized with heart failure. Am Heart J. 2005;150(5):982

13. Kripalani S, Roumie CL, Dalal AK, et al. Effect of a pharmacist intervention on clinically important medication errors after hospital discharge: a randomized trial. Ann Intern Med. 2012;157(1):1-10.

14. Gil M, Mikaitis DK, Shier G, Johnson TJ, Sims S. Impact of a combined pharmacist and social worker program to reduce hospital readmissions. J Manag Care Pharm. 2013;19(7):558-63. Available at: http://www.amcp.org/ JMCP/2013/September_2013/17104/1033.html.

15. Cavanaugh JJ, Lindsey KN, Shilliday BB, Ratner SP. Pharmacistcoordinated multidisciplinary hospital follow-up visits improve patient outcomes. J Manag Care Spec Pharm. 2015;21(3):256-60. Available at: http:// www.amcp.org/JMCP/2015/March/19115/1033.html.

16. Kwan JL, Lo L, Sampson M, Shojania KG. Medication reconciliation during transitions of care as a patient safety strategy: a systematic review. Ann Intern Med. 2013;158(5):397-403.

17. Wiggins BS, Rodgers JE, DiDomenico RJ, Cook AM, Page RL 2nd. Discharge counseling for patients with heart failure or myocardial infarction: a best practices model developed by members of the american college of clinical pharmacy's cardiology practice and research network based on the hospital to home (H2H) initiative. Pharmacotherapy. 2013;33(5):558-80.

18. Kilcup M, Schultz D, Carlson J, Wilson B. Postdischarge pharmacist medication reconciliation: impact on readmission rates and financial savings. J Am Pharm Assoc (2003). 2013;53(1):78-84.

19. Kitts NK, Reeve AR, Tsu L. Care transitions in elderly heart failure patients: current practices and the pharmacist's role. Consult Pharm. 2014:29(3):179-90.

20. Ponniah A, Anderson B, Shakib S, Doecke CJ, Angley M. Pharmacists' role in the post-discharge management of patients with heart failure: a literature review. J Clin Pharm Ther. 2007;32(4):343-52.

21. Kaboli PJ, Hoth AB, McClimon BJ, Schnipper JL. Clinical pharmacists and inpatient medical care: a systematic review. Arch Intern Med. 2006;166(9):955-64.

22. Hesselink G, Schoonhoven L, Barach P, et al. Improving patient handovers from hospital to primary care: a systematic review. Ann Intern Med. 2012;157(6):417-28.

23. Thomas R, Huntley AL, Mann M, et al. Pharmacist-led interventions to reduce unplanned admissions for older people: a systematic review and meta-analysis of randomised controlled trials. Age Ageing. 2014:43(2):174-87.
24. Hansen LO, Young RS, Hinami K, Leung A, Williams MV. Interventions to reduce 30-day rehospitalization: a systematic review. Ann Intern Med. 2011;155(8):520-28

25. Royal S, Smeaton L, Avery AJ, Hurwitz B, Sheikh A. Interventions in primary care to reduce medication related adverse events and hospital admissions: systematic review and meta-analysis. Qual Saf Health Care. 2006;15(1):23-31.

26. Christensen M, Lundh A. Medication review in hospitalised patients to reduce morbidity and mortality. Cochrane Database Syst Rev. 2013;2:CD008986.

27. Graabaek T, Kjeldsen LJ. Medication reviews by clinical pharmacists at hospitals lead to improved patient outcomes: a systematic review. Basic Clin Pharmacol Toxicol. 2013;112(6):359-73.

28. Lehnbom EC, Stewart MJ, Manias E, Westbrook JI. Impact of medication reconciliation and review on clinical outcomes. Ann Pharmacother. 2014;48(10):1298-312.

29. Okumura LM, Rotta I, Correr CJ. Assessment of pharmacist-led patient counseling in randomized controlled trials: a systematic review. Int J Clin Pharm. 2014;36(5):882-91.

30. Moher D, Liberati A, Tetzlaff J, Altman DG; PRISMA Group. Preferred reporting items for systematic reviews and meta-analyses: the PRISMA statement. PLoS Med. 2009;6(7):e1000097.

31. Higgins JP, Altman DG, Gøtzsche PC, et al. The Cochrane Collaboration's tool for assessing risk of bias in randomised trials. BMJ. 2011;343:d5928.

32. Furlan AD, Pennick V, Bombardier C, van Tulder M; Editorial Board, Cochrane Back Review Group. 2009 updated method guidelines for systematic reviews in the Cochrane Back Review Group. Spine (Phila Pa 1976). 2009;34(18):1929-1941.

33. O'Rourke K., Detsky AS. Meta-analysis in medical research: strong encouragement for higher quality in individual research efforts. J Clin Epidemiol. 1989;42(10):1021-24.

34. Treadwell JR, Singh S, Talati R, McPheeters ML, Reston JT. A framework for best evidence approaches can improve the transparency of systematic reviews. J Clin Epidemiol. 2012;65(11):1159-62.

35. van Tulder M, Furlan A, Bombardier C, Bouter L; Editorial Board, Cochrane Collaboration Back Review Group. Updated method guidelines for systematic reviews in the Cochrane Collaboration Back Review Group. Spine (Phila Pa 1976). 2003;28(12):1290-99.

36. Dudas V, Bookwalter T, Kerr KM, Pantilat SZ. The impact of follow-up telephone calls to patients after hospitalization. Am J Med. 2001;111(9B):26S-30S.

37. Barker A, Barlis P, Berlowitz D, Page K, Jackson B, Lim WK. Pharmacist directed home medication reviews in patients with chronic heart failure: a randomised clinical trial. Int J Cardiol. 2012;159(2):139-43.

38. Bolas H, Brookes K, Scott M, McElnay J. Evaluation of a hospital-based community liaison pharmacy service in Northern Ireland. Pharm World Sci. 2004;26(2):114-20.

39. Holland R, Lenaghan E, Harvey I, et al. Does home based medication review keep older people out of hospital? The HOMER randomised controlled trial. BMJ. 2005;330(7486):293.

40. Holland R, Brooksby I, Lenaghan E, et al. Effectiveness of visits from community pharmacists for patients with heart failure: HeartMed randomised controlled trial. BMJ. 2007;334(7603):1098.

41. Jack BW, Chetty VK, Anthony D, et al. A reengineered hospital discharge program to decrease rehospitalization: a randomized trial. Ann Intern Med. 2009;150(3):178-87.

42. Koehler BE, Richter KM, Youngblood L, et al. Reduction of 30-day postdischarge hospital readmission or emergency department (ED) visit rates in high-risk elderly medical patients through delivery of a targeted care bundle. J Hosp Med. 2009;4(4):211-18. 
43. Lipton HL, Bird JA. The impact of clinical pharmacists' consultations on geriatric patients' compliance and medical care use: a randomized controlled trial. Gerontologist. 1994;34(3):307-15.

44. Lisby M, Thomsen A, Nielsen LP, et al. The effect of systematic medication review in elderly patients admitted to an acute ward of internal medicine. Basic Clin Pharmacol Toxicol. 2010;106(5):422-27.

45. López Cabezas C, Falces Salvador C, Cubi Quadrada D, et al. Randomized clinical trial of a postdischarge pharmaceutical care program vs regular follow-up in patients with heart failure. Farm Hosp. 2006;30(6):328-42.

46. Makowsky MJ, Koshman SL, Midodzi WK, Tsuyuki RT. Capturing outcomes of clinical activities performed by a rounding pharmacist practicing in a team environment: the COLLABORATE study [NCT00351676]. Med Care. 2009; 47(6):642-50.

47. Naunton M, Peterson GM. Evaluation of home-based follow-up of highrisk elderly patients discharged from hospital. J Pharm Res. 2003;33(3):176-82.

48. Nazareth I, Burton A, Shulman S, Smith P, Haines A, Timberal H. A pharmacy discharge plan for hospitalized elderly patients-a randomized controlled trial. Age Ageing. 2001;30(1):33-40.

49. Rainville EC. Impact of pharmacist interventions on hospital readmissions for heart failure. Am J Health Syst Pharm. 1999;56(13):1339-42.

50. Schmader KE, Hanlon JT, Pieper CF, et al. Effects of geriatric evaluation and management on adverse drug reactions and suboptimal prescribing in the frail elderly. Am J Med. 2004;116(6):394-401.

51. Schnipper JL, Kirwin JL, Cotugno MC, et al. Role of pharmacist counseling in preventing adverse drug events after hospitalization. Arch Intern Med. 2006;166(5):565-71

52. Schnipper JL, Hamann C, Ndumele CD, et al. Effect of an electronic medication reconciliation application and process redesign on potential adverse drug events: a cluster-randomized trial. Arch Intern Med. 2009;169(8):771-80.

53. Scullin C, Scott MG, Hogg A, McElnay JC. An innovative approach to integrated medicines management. J Eval Clin Pract. 2007;13(5):781-88.

54. Stewart S, Pearson S, Horowitz JD. Effects of a home-based intervention among patients with congestive heart failure discharged from acute hospital care. Arch Intern Med. 1998;158(10):1067-72.

55. Stewart S, Pearson S, Luke CG, Horowitz JD. Effects of home-based intervention on unplanned readmissions and out-of-hospital deaths. J Am Geriatr Soc. 1998;46(2):174-80.
56. Stowasser DA, Collins DM, Stowasser M. A randomised controlled trial of medication liaison services - patient outcomes. J Pharm Pract Res. 2002;32(2):133-40.

57. Triller DM, Hamilton RA. Effect of pharmaceutical care services on outcomes for home care patients with heart failure. Am J Health Syst Pharm. 2007;64(21):2244-49.

58. Al-Rashed SA, Wright DJ, Roebuck N, Sunter W, Chrystyn H. The value of inpatient pharmaceutical counselling to elderly patients prior to discharge. Br J Clin Pharmacol. 2002;54(6):657-64.

59. Farris KB, Carter BL, Xu Y, et al. Effect of a care transition intervention by pharmacists: an RCT. BMC Health Serv Res. 2014;14(1):406.

60. Hawes EM, Maxwell WD, White SF, Mangun J, Lin FC. Impact of an outpatient pharmacist intervention on medication discrepancies and health care resource utilization in posthospitalization care transitions. J Prim Care Community Health. 2014;5(1):14-18.

61. Salter C, Holland R, Harvey I, Henwood K. "I haven't even phoned my doctor yet." The advice giving role of the pharmacist during consultations for medication review with patients aged 80 or more: qualitative discourse analysis. BMJ. 2007;334(7603):1101.

62. Boutwell A, Hwu S. Effective Interventions to Reduce Rehospitalizations: A Survey of the Published Evidence. Cambridge, MA: Institute for Healthcare Improvement; 2009.

63. Cawthon C, Walia S, Osborn CY, Niesner KJ, Schnipper JL, Kripalani S. Improving care transitions: the patient perspective. J Health Commun. 2012;17(Suppl 3):312-24.

64. Hatah E, Braund R, Tordoff J, Duffull SB. A systematic review and meta-analysis of pharmacist-led fee-for-services medication review. Br J Clin Pharmacol. 2014;77(1):102-15.

65. Shekelle PG, Wachter RM, Pronovost PJ, et al. Making health care safer II: an updated critical analysis of the evidence for patient safety practices. Evid Rep Technol Assess (Full Rep). 2013;(211):1-945.

66. Giberson S, Yoder S, Lee MP. Improving patient and health system outcomes through advanced pharmacy practice: a report to the U.S. Surgeon General. U.S. Public Health Service. December 2011. Available at: http:// www.accp.com/docs/positions/misc/improving_patient_and_health_system_outcomes.pdf. Accessed June 8, 2015. 


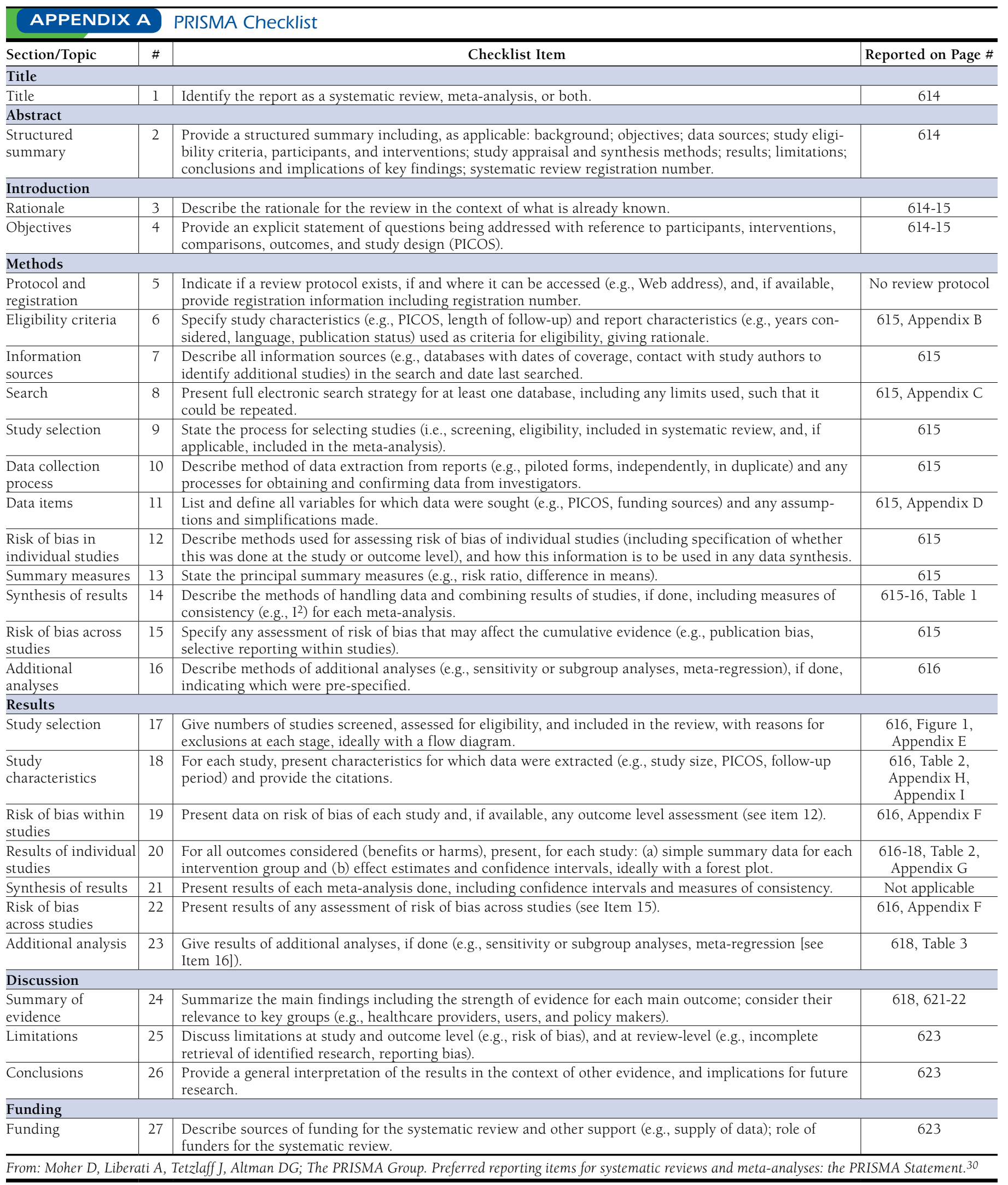


Identifying the Optimal Role for Pharmacists in Care Transitions: A Systematic Review

APPENDIX B Inclusion and Exclusion Criteria According to PICO for Systematic Review

\begin{tabular}{|c|c|}
\hline \multicolumn{2}{|r|}{ Inclusion Criteria } \\
\hline Study design & - (quasi) (cluster) Randomized controlled trial published in English \\
\hline Population & - Adult participants admitted to a hospital and discharged home \\
\hline Setting & - Intervention conducted in hospital and/or community pharmacy and/or patients' home \\
\hline Intervention & $\begin{array}{l}\text { - The intervention involved a pharmacist, pregraduate pharmacist, or pharmacy technician } \\
\text { - The pharmacist had a proactive role, meaning for all interventions, patients received an active pharmacist intervention (e.g., } \\
\text { excluding reactively responding to physician questions during ward rounds) } \\
\text { - Interventions were performed before, during, or up to } 30 \text { days after hospitalization. The postdischarge time limit was chosen to } \\
\text { ensure connection to transitional care } \\
\text { - The intervention was designed to improve transitional care and aimed at medication-related issues }\end{array}$ \\
\hline Comparison & - The intervention was compared with a control group that received usual care \\
\hline Clinical outcomes & $\begin{array}{l}\text { - At least } 1 \text { of the following outcomes was measured: mortality, readmissions, emergency department visits, and adverse drug } \\
\text { events }\end{array}$ \\
\hline \multicolumn{2}{|r|}{ Exclusion Criteria } \\
\hline Participant & - Intervention conducted solely on pediatric patients or psychiatric patients due to their specific population characteristics \\
\hline Setting & - Interventions in a palliative care setting or in an intensive care ward due to their specific setting characteristics \\
\hline Intervention & $\begin{array}{l}\text { - Interventions solely targeted at specific drugs (e.g., improving adherence of statins) } \\
\text { - Interventions not aimed at transitional care (e.g., interventions in outpatient clinics without transmission of relevant information } \\
\text { from earlier health care provider encounters in the hospital or interventions on heart failure guideline adherence) }\end{array}$ \\
\hline
\end{tabular}

\section{APPENDIX C Search Strategy}

\section{MEDLINE}

("patient admission"[mesh] OR "patient admission" OR "admission"[TIAB] OR "hospital admission"[TIAB] OR "hospitalization"[mesh] OR "patient discharge"[mesh] OR "discharge"[TIAB] OR "discharged"[TIAB] OR "post discharge"[TIAB] OR "postdischarge"[TIAB] OR "hospitals"[mesh] OR "hospital setting"[TIAB]) AND ("patient education as topic"[mesh] OR "counseling"[mesh] OR "counselling"[TIAB] OR "medication counseling" [TIAB] OR "knowledge"[TIAB] OR "drug knowledge"[TIAB] OR "medicines knowledge"[TIAB] OR "medication knowledge"[TIAB] OR "education" [TIAB] OR "medication understanding"[TIAB] OR "Medication Therapy Management"[mesh] OR "integrated medicines management"[TIAB] OR "medicine* management"[TIAB] OR "drug* management"[TIAB] OR "Medication Errors/ prevention and control"[Mesh] OR "medication reconciliation"[mesh] OR "medication reconciliation"[TIAB] OR "medical history taking"[mesh] OR "medication history taking"[TIAB] OR "medication history"[TIAB] OR "medicines histories"[TIAB] OR "continuity of patient care"[mesh] OR "Patient Care Planning"[mesh] OR "discharge planning" [TIAB] OR "discharge service"[TIAB] OR "discharge booklet"[TIAB] OR "follow-up"[TIAB] OR "follow up"[TIAB] OR transition*[TIAB] OR "seamless" [TIAB] OR "care bundle"[TIAB] OR "care coordination" [TIAB] OR "aftercare"[mesh] OR "aftercare"[TIAB] OR "continuity"[TIAB] OR "Outreach"[TIAB] OR "co-ordination" [TIAB] OR "coordination" [TIAB] OR "house calls"[mesh] OR "home visit*"[TIAB] OR "house visit*"[TIAB] OR "pharmacy visit"[TIAB] OR "pharmacist visit"[TIAB] OR "home based intervention"[TIAB] OR "telephone call*"[TIAB] OR "telephone"[TIAB] OR "phone call*"[TIAB] OR "phone"[TIAB] OR "medication review" [tw] OR "drug utilization review"[mesh] OR "treatment review"[TIAB] OR "medication review"[TIAB] OR "medicines review"[TIAB] OR "drug review" [TIAB] OR "reviewing medication"[TIAB] OR "monitoring pharmacotherapy"[TIAB] OR "optimizing drug regimens"[TIAB] OR "pharmaceutical care"[TIAB]) AND ("community pharmacy services"[mesh] OR "Pharmacy Service, Hospital"[mesh] OR "pharmaceutical preparations"[mesh] OR "drug prescriptions"[mesh] OR "pharmacists" OR "pharmacist*"OR "pharmacists"[mesh] OR "pharmacy"[TIAB] OR "pharmacist"[TIAB] OR "pharmacists"[TIAB] OR "pharmaceutical"[TIAB] OR "Pharmacists' Aides" [TIAB] OR "pharmacy technician"[TIAB] OR "pharmacy practitioners"[TIAB] OR pharmacy service[TIAB] OR "medication"[TIAB] OR "medication liaison services"[TIAB]) AND (randomized controlled trial[Publication Type] OR (randomized[Title/Abstract] AND controlled[Title/Abstract] AND trial[Title/ Abstract]) OR (randomised[Title/Abstract] AND controlled[Title/Abstract] AND trial[Title/Abstract]) OR (random*[Title/Abstract]))

(Limits: English)

\section{EMBASE}

\#l= 'hospital admission'/de OR 'patient admission' OR 'admission' OR 'hospital admission' OR 'hospitalization'/de OR 'hospital discharge'/de OR 'discharge' OR 'discharged' OR 'post discharge' OR 'postdischarge' OR 'hospital'/de OR 'hospital setting'

\#2= 'patient education'/de OR 'counseling'/de OR 'counselling' OR 'medication counseling' OR 'knowledge' OR 'drug knowledge' OR 'medicines knowledge' OR 'medication knowledge' OR 'education' OR 'medication understanding' OR 'medication therapy management'/de OR 'integrated medicines management' OR 'medicine management' OR 'medicines management' OR 'drug management' OR 'drugs management' OR 'medication reconciliation' OR 'medication error'/de OR 'anamnesis'/de OR 'medication history taking' OR 'medication history' OR 'medicines histories' OR 'patient care'/de OR 'patient care planning'/de OR 'discharge planning' OR 'discharge service' OR 'discharge booklet' OR 'follow-up' OR 'follow up' OR 'transition' OR 'seamless' OR 'care bundle' OR 'care coordination' OR 'aftercare'/de OR 'aftercare' OR 'continuity' OR 'outreach' OR 'co-ordination' OR 'coordination' OR 'professional practice'/de OR 'house calls' OR 'home visit' OR 'home visits' OR 'house visit' OR 'house visits' OR 'pharmacy visit' OR 'pharmacist visit' OR 'home based intervention' OR 'telephone call' OR 'telephone calls' OR 'telephone' OR 'phone call' OR 'phone calls' OR 'phone' OR 'pharmaceutical care'/de OR 'drug utilization review' OR 'treatment review' OR 'medication review' OR 'medicines review' OR 'drug review' OR 'reviewing medication' OR 'monitoring pharmacotherapy' OR 'optimizing drug regimens' OR 'pharmaceutical care'

\#3= 'pharmacy'/de OR 'hospital pharmacy'/de OR 'drug'/de OR 'prescription'/de OR 'pharmacist'/de OR 'pharmacy technician'/de OR 'drug therapy'/de OR 'pharmacy' OR 'pharmacist' OR 'pharmacists' OR 'pharmaceutical' OR 'pharmacists aides' OR 'pharmacy technician' OR 'pharmacy practitioners' OR 'pharmacy service' OR 'medication' OR 'medication liaison services' 


\section{APPENDIX C Search Strategy (continued)}

\#4= 'randomized controlled trial'/de OR 'randomized controlled trial (topic)'/de OR (randomized AND controlled AND trial)

\#5= \#1 AND \#2 AND \#3 AND \#4

(Limits: English AND human studies AND EMBASE)

\section{International Pharmaceutical Abstracts}

\#l= TI ( "patient admission" OR "admission" OR "hospital admission" OR "hospitalization" OR "patient discharge" OR "discharge" OR "discharged" OR "post discharge" OR "postdischarge" OR "hospitals" OR "hospital setting") OR AB ( "patient admission" OR "admission" OR "hospital admission" OR "hospitalization" OR "patient discharge" OR "discharge" OR "discharged" OR "post discharge" OR "postdischarge" OR "hospitals" OR "hospital setting" )

\#2= TI ( "patient education" OR "counseling" OR "counselling" OR "medication counseling" OR "knowledge" OR "drug knowledge" OR "medicines knowledge" OR "medication knowledge" OR "education" OR "medication understanding" OR "Medication Therapy Management" OR "integrated medicines management" OR "medicine* management" OR "drug* management" OR "Medication Errors" OR "medication reconciliation" OR "medication reconciliation" OR "medical history taking" OR "medication history" OR "medicines histories" OR "continuity of patient care" OR "Patient Care Planning" OR "discharge planning" OR "discharge service" OR "discharge booklet" OR "follow-up" OR "follow up" OR transition* OR "seamless" OR "care bundle" OR "care coordination" OR "aftercare" OR "continuity" OR "outreach" OR "co-ordination" OR "coordination" OR "house calls" OR "home visit*" OR "house visit*" OR "pharmacy visit" OR "pharmacist visit" OR "home based intervention" OR "telephone call" OR "telephone" OR "phone call*" OR "phone" OR "medication review" OR "drug utilization review" OR "treatment review" OR "medication review" OR "medicines review" OR "drug review" OR "reviewing medication" OR "monitoring pharmacotherapy" OR "optimizing drug regimens" OR "pharmaceutical care") OR AB ( "patient education" OR "counseling" OR "counselling" OR "medication counseling" OR "knowledge" OR "drug knowledge" OR "medicines knowledge" OR "medication knowledge" OR "education" OR "medication understanding" OR "Medication Therapy Management" OR "integrated medicines management" OR "medicine* management" OR "drug* management" OR "Medication Errors" OR "medication reconciliation" OR "medication reconciliation" OR "medical history taking" OR "medication history" OR "medicines histories" OR "continuity of patient care" OR "Patient Care Planning" OR "discharge planning" OR "discharge service" OR "discharge booklet" OR "follow-up" OR "follow up" OR transition* OR "seamless" OR "care bundle" OR "care coordination" OR "aftercare" OR "continuity" OR "outreach" OR "co-ordination" OR "coordination" OR "house calls" OR "home visit*" OR "house visit*" OR "pharmacy visit" OR "pharmacist visit" OR "home based intervention" OR "telephone call*" OR "telephone" OR "phone call*" OR "phone" OR "medication review" OR "drug utilization review" OR "treatment review" OR "medication review" OR "medicines review" OR "drug review" OR "reviewing medication" OR "monitoring pharmacotherapy" OR "optimizing drug regimens" OR "pharmaceutical care" )

\#3= TI ( "community pharmacy services" OR "Hospital Pharmacy Service" OR "pharmaceutical preparations" OR "drug prescriptions" OR "pharmacist*" OR "pharmacy" OR "pharmaceutical" OR "Pharmacists' Aides" OR "pharmacy technician" OR "pharmacy practitioners" OR "pharmacy service" OR "medication" OR "medication liaison services") OR AB ( "community pharmacy services" OR "Hospital Pharmacy Service" OR "pharmaceutical preparations" OR "drug prescriptions" OR "pharmacist*" OR "pharmacy" OR "pharmaceutical" OR "Pharmacists' Aides" OR "pharmacy technician" OR "pharmacy practitioners" OR "pharmacy service" OR "medication" OR "medication liaison services" )

\#4= TI ( "randomized controlled trial" OR (randomized AND controlled AND trial) OR (randomised AND controlled AND trial) OR random*) OR AB ( "randomized controlled trial" OR (randomized AND controlled AND trial) OR (randomised AND controlled AND trial) OR random*)

\#5= \#1 AND \#2 AND \#3 AND \#4

(Limits: English AND human studies) 


\section{APPENDIX D List of Extracted Parameters}

\begin{tabular}{|c|c|c|}
\hline Cluster & Data Abbreviation & Data Description \\
\hline \multirow[t]{4}{*}{ Study reference } & Author & Primary author of reference \\
\hline & Full title & Full title \\
\hline & YoP & Year of publication \\
\hline & YoE & Year of execution \\
\hline \multirow[t]{9}{*}{ Study design } & Design & Design of study \\
\hline & \multirow[t]{4}{*}{ Specific setting of study } & Multi/single center \\
\hline & & Type of hospital \\
\hline & & Number of beds \\
\hline & & Specific wards? \\
\hline & Country & Country where study was performed \\
\hline & Funding & Was the study funded? \\
\hline & C.o.I. & Have the authors reported a conflict of interest? \\
\hline & Objective & Description of the main objective of the conducted study \\
\hline \multirow[t]{14}{*}{ Method } & Inclusion criteria & All inclusion criteria as reported in original study \\
\hline & Exclusion criteria & All exclusion criteria as reported in original study \\
\hline & Usual care & Description of usual care \\
\hline & Years of experience & Years of experience from the performer \\
\hline & Protocol training & What kind of training was received by personnel performing the intervention? \\
\hline & According to protocol & Did the personnel have protocols to perform the intervention? \\
\hline & \multirow{3}{*}{$\begin{array}{l}\text { Who is collaborating with the } \\
\text { pharmacist? }\end{array}$} & Who: Which other health care providers performed an intervention? \\
\hline & & What: Describe the intervention of the OTHER health care provider \\
\hline & & Communication: How did they communicate? \\
\hline & Access to medical information & Does the performing pharmacist have access to medical information and in what way? \\
\hline & \multirow[t]{4}{*}{ Pharmacy intervention } & When: At what time during hospitalization is the pharmacy intervention performed? \\
\hline & & What: Description of intervention performed \\
\hline & & Who: Which member of the pharmacy staff is performing the intervention? \\
\hline & & Time spent: Total time spent of intervention \\
\hline \multirow{16}{*}{$\begin{array}{l}\text { Patient } \\
\text { characteristics }\end{array}$} & Total Nr. assessed & Number of patients that are assessed for eligibility in the study \\
\hline & Total Nr. randomized & Number of patients that are randomized in the study \\
\hline & \multirow{2}{*}{$\begin{array}{l}\text { Significant differences at } \\
\text { baseline }\end{array}$} & Are there significant differences at baseline? \\
\hline & & Describe the differences \\
\hline & Nr. randomized patients: I and C & Number of patients in intervention group and control group after randomization \\
\hline & Male \% & Percentage of men in study \\
\hline & Age: I and $\mathrm{C}$ & Mean age, range and standard deviation of intervention and control groups \\
\hline & \multirow[t]{3}{*}{ Health state } & Main: Primary diagnosis of population group (e.g., heart failure) \\
\hline & & Total number of comorbidities \\
\hline & & How are the total number of comorbidities measured? \\
\hline & \multirow[t]{2}{*}{ Nr. medication } & When: At what time during hospitalization? \\
\hline & & Mean number, range, and standard deviation of medication in intervention and control groups \\
\hline & \% living alone: I and $\mathrm{C}$ & Percentage living alone in intervention and control groups \\
\hline & \multirow[t]{2}{*}{ Nr of hospitalizations } & Mean number of previous hospitalizations in intervention and control groups \\
\hline & & Time: during which time frame? \\
\hline & Other demographic information & If mentioned in study, for example, education, ethnicity, social class \\
\hline \multirow[t]{10}{*}{ Outcomes } & \multirow[t]{6}{*}{ Outcomes } & Outcome in short \\
\hline & & Primary outcome \\
\hline & & Definition: report the outcome as described by authors \\
\hline & & Method: how is the outcome measured? \\
\hline & & When: when is the outcome measured (=endpoint)? \\
\hline & & What: what is exactly measured? \\
\hline & \multirow[t]{3}{*}{ Nr. of patients analyzed } & Number of patients in intervention group and control group for analysis \\
\hline & & Characteristics of the (different) outcomes \\
\hline & & Statistics of the outcomes \\
\hline & Other outcomes & Definition, method, endpoint of measurement, percentages, significance \\
\hline \multirow[t]{3}{*}{ Other } & Limitations & Limitations as mentioned by the authors \\
\hline & Conclusion & Conclusion as mentioned by the authors \\
\hline & Comments & Relevant/notable comments \\
\hline \multirow[t]{3}{*}{ Reviewer } & Name & Clementine Stuijt or Rik Ensing \\
\hline & Date & Date of data extraction \\
\hline & Check & Checked by second reviewer: type name \\
\hline
\end{tabular}


APPENDIX E Inter-Rater Agreement Calculation for Full-Text Assessment

\begin{tabular}{|c|c|c|c|c|}
\hline & & \multicolumn{2}{|c|}{ HE } & \\
\hline & & $\mathrm{N}$ & $\mathrm{Y}$ & Total \\
\hline \multirow{2}{*}{$\mathrm{CS}$} & $\mathrm{N}$ & 90 & 6 & 96 \\
\cline { 2 - 5 } & $\mathrm{Y}$ & 2 & 28 & 30 \\
\hline \multicolumn{2}{|c|}{ CS=author/reviewer Clementine C.M. Stuijt; HE=author/reviewer Hendrik T. Ensing; $N=$ no; $Y=$ yes. } \\
\hline
\end{tabular}

Observed similarity: $((90+28) / 126) \times 100=94 \%$

Kappa calculation formula:

$$
\text { - } \kappa=\frac{(\operatorname{Pr}(a)-\operatorname{Pr}(e)}{1-\operatorname{Pr}(e)}
$$

In which:

$\operatorname{Pr}(a)=$ relative observed agreement among raters $=(90+28) / 126=0.94$

To calculate $\operatorname{Pr}(e)$ (the probability of random agreement):

- Ensing said "Y" to 34 articles and "N" to 92 articles: Thus "Y" is 0.37 of the time.

- Stuijt said "Y" to 30 articles and "N" to 96 articles. Thus "Y" is 0.31 of the time.

- The probability that both say "Y" is $0.37 \times 0.31=0.11$.

- The probability that both say "N" is $(1-0.37) \times(1-0.31)=0.43$.

- Thus the overall probability of random agreement is $\operatorname{Pr}(e)=0.11+0.43=0.54$.

Resulting in:

- $\kappa=\frac{0.94-0.54}{1-0.54}=0.87(0.81-1$ is almost perfect $)$ 


\begin{tabular}{|c|c|c|c|}
\hline Primary Author & Primary Title & \begin{tabular}{|c|}
$\begin{array}{c}\text { Publication } \\
\text { Year }\end{array}$ \\
\end{tabular} & $\begin{array}{l}\text { Reason for } \\
\text { Exclusion }\end{array}$ \\
\hline Adepu, R & Influence of postdischarge counselling on health outcomes in diabetic and hypertensive patients & 2011 & Study design \\
\hline Ahmed, A & $\begin{array}{l}\text { Quality and outcomes of heart failure care in older adults: role of multidisciplinary disease-man- } \\
\text { agement programs }\end{array}$ & 2002 & Study design \\
\hline Allen, K & $\begin{array}{l}\text { A randomized trial testing the superiority of a postdischarge care management model for stroke } \\
\text { survivors }\end{array}$ & 2009 & $\begin{array}{l}\text { No pharmacist } \\
\text { intervention }\end{array}$ \\
\hline Anderegg, S & $\begin{array}{l}\text { Acceptance of recommendations by inpatient pharmacy case managers: unintended consequences } \\
\text { of hospitalist and specialist care }\end{array}$ & 2013 & Study design \\
\hline Backes, A & Primary medication adherence among patients transitioning from hospital to home care & 2012 & $\begin{array}{l}\text { Meeting report or } \\
\text { editorial }\end{array}$ \\
\hline Baker, D & Evaluation of drug information for cardiology patients & 1991 & $\begin{array}{l}\text { No pharmacist } \\
\text { intervention }\end{array}$ \\
\hline Baker, DM & A study contrasting different modalities of medication discharge counseling & 1984 & $\begin{array}{l}\text { Outcome not } \\
\text { included in review }\end{array}$ \\
\hline Basoor, A & Result of quality improvement discharge tool in congestive heart failure-randomized controlled trial & 2011 & Study design \\
\hline Becerra-Camargo, J & $\begin{array}{l}\text { A multicentre, double-blind, randomised, controlled, parallel-group study of the effectiveness of a } \\
\text { pharmacist-acquired medication history in an emergency department }\end{array}$ & 2013 & $\begin{array}{l}\text { Outcome not } \\
\text { included in review }\end{array}$ \\
\hline Beckett, RD & $\begin{array}{l}\text { Effectiveness and feasibility of pharmacist-led admission medication reconciliation for geriatric } \\
\text { patients }\end{array}$ & 2012 & $\begin{array}{l}\text { Outcome not } \\
\text { included in review }\end{array}$ \\
\hline Beney, J & $\begin{array}{l}\text { Effect of telephone follow-up on the physical well-being dimension of quality of life in patients } \\
\text { with cancer }\end{array}$ & 2002 & $\begin{array}{l}\text { Outcome not } \\
\text { included in review }\end{array}$ \\
\hline Bladh, L & $\begin{array}{l}\text { Effects of a clinical pharmacist service on health-related quality of life and prescribing of drugs: a } \\
\text { randomised controlled trial }\end{array}$ & 2011 & $\begin{array}{l}\text { Outcome not } \\
\text { included in review }\end{array}$ \\
\hline Blix, HS & $\begin{array}{l}\text { Characteristics of drug-related problems discussed by hospital pharmacists in multidisciplinary } \\
\text { teams }\end{array}$ & 2006 & Study design \\
\hline Bollella, G & $\begin{array}{l}\text { Optimal level of liaison pharmacist intervention to facilitate a post-discharge home medicines } \\
\text { review }\end{array}$ & 2008 & $\begin{array}{l}\text { Outcome not } \\
\text { included in review }\end{array}$ \\
\hline Bonnet-Zamponi, D & $\begin{array}{l}\text { Drug-related readmissions to medical units of older adults discharged from acute geriatric units: } \\
\text { results of the Optimization of Medication in AGEd multicenter randomized controlled trial }\end{array}$ & 2013 & $\begin{array}{l}\text { No pharmacist } \\
\text { intervention }\end{array}$ \\
\hline Brullet, E & $\begin{array}{l}\text { A randomized study of the safety of outpatient care for patients with bleeding peptic ulcer treated } \\
\text { by endoscopic injection }\end{array}$ & 2004 & $\begin{array}{l}\text { No pharmacist } \\
\text { intervention }\end{array}$ \\
\hline Burnett, KM & $\begin{array}{l}\text { Effects of an integrated medicines management program on medication appropriateness in hospi- } \\
\text { talized patients }\end{array}$ & 2009 & $\begin{array}{l}\text { Outcome not } \\
\text { included in review }\end{array}$ \\
\hline Calvert, SB & $\begin{array}{l}\text { Patient-focused intervention to improve long-term adherence to evidence-based medications: a } \\
\text { randomized trial }\end{array}$ & 2012 & $\begin{array}{l}\text { Outcome not } \\
\text { included in review }\end{array}$ \\
\hline Cannon, J & $\begin{array}{l}\text { Pharmaceutical care provision to elderly patients: assessment of its impact on compliance and } \\
\text { discharge medication changes }\end{array}$ & 1999 & $\begin{array}{l}\text { Outcome not } \\
\text { included in review }\end{array}$ \\
\hline Cawthon, C & Improving care transitions: the patient perspective & 2012 & Study design \\
\hline Ching, CL & $\begin{array}{l}\text { Impact of pharmaceutical care on readmission rates and quality of life in coronary artery disease } \\
\text { patients }\end{array}$ & 2002 & $\begin{array}{l}\text { Meeting report or } \\
\text { editorial }\end{array}$ \\
\hline Connor, $\mathrm{MO}$ & Prevention of adverse drug events in hospitalised older patients: a randomised controlled trial & 2012 & $\begin{array}{l}\text { Meeting report or } \\
\text { editorial }\end{array}$ \\
\hline Cordasco, KM & A low-literacy medication education tool for safety-net hospital patients & 2009 & $\begin{array}{l}\text { No pharmacist } \\
\text { intervention }\end{array}$ \\
\hline Davidson, J & Pre-discharge counseling in the elderly: what difference does it make? & 1989 & $\begin{array}{l}\text { Outcome not } \\
\text { included in review }\end{array}$ \\
\hline de Wit, R & $\begin{array}{l}\text { Improving the quality of pain treatment by a tailored pain education programme for cancer } \\
\text { patients in chronic pain }\end{array}$ & 2001 & $\begin{array}{l}\text { No pharmacist } \\
\text { intervention }\end{array}$ \\
\hline Doughty, RN & $\begin{array}{l}\text { Randomized, controlled trial of integrated heart failure management: the Auckland Heart Failure } \\
\text { Management Study }\end{array}$ & 2002 & $\begin{array}{l}\text { No pharmacist } \\
\text { intervention }\end{array}$ \\
\hline Dromerick, AW & $\begin{array}{l}\text { Preventing recurrence of thromboembolic events through coordinated treatment in the District of } \\
\text { Columbia }\end{array}$ & 2011 & $\begin{array}{l}\text { No pharmacist } \\
\text { intervention }\end{array}$ \\
\hline Eggink, RN & $\begin{array}{l}\text { The effect of a clinical pharmacist discharge service on medication discrepancies in patients with } \\
\text { heart failure }\end{array}$ & 2010 & $\begin{array}{l}\text { Outcome not } \\
\text { included in review }\end{array}$ \\
\hline Esposito, L & The effects of medication education on adherence to medication regimens in an elderly population & 1995 & $\begin{array}{l}\text { No pharmacist } \\
\text { intervention }\end{array}$ \\
\hline Ferrante, D & $\begin{array}{l}\text { Long-term results after a telephone intervention in chronic heart failure: DIAL (Randomized Trial } \\
\text { of Phone Intervention in Chronic Heart Failure) follow-up }\end{array}$ & 2010 & $\begin{array}{l}\text { No pharmacist } \\
\text { intervention }\end{array}$ \\
\hline Gallagher, PF & $\begin{array}{l}\text { Prevention of potentially inappropriate prescribing for elderly patients: a randomized controlled } \\
\text { trial using STOPP/START criteria }\end{array}$ & 2011 & $\begin{array}{l}\text { No pharmacist } \\
\text { intervention }\end{array}$ \\
\hline
\end{tabular}




\begin{tabular}{|c|c|c|c|}
\hline Primary Author & Primary Title & $\begin{array}{c}\text { Publication } \\
\text { Year }\end{array}$ & $\begin{array}{l}\text { Reason for } \\
\text { Exclusion }\end{array}$ \\
\hline Gattis, WA & $\begin{array}{l}\text { Reduction in heart failure events by the addition of a clinical pharmacist to the heart failure } \\
\text { management team: results of the Pharmacist in Heart Failure Assessment Recommendation and } \\
\text { Monitoring (PHARM) Study }\end{array}$ & 1999 & No transitional care \\
\hline George, L & $\begin{array}{l}\text { Impact of a surgical preadmission clinic pharmacist on the quality of medication management } \\
\text { from preadmission to discharge: a randomised controlled study }\end{array}$ & 2011 & $\begin{array}{l}\text { Outcome not } \\
\text { included in review }\end{array}$ \\
\hline Gizzi, LA & $\begin{array}{l}\text { Assessment of a safety enhancement to the hospital medication reconciliation process for elderly } \\
\text { patients }\end{array}$ & 2010 & $\begin{array}{l}\text { Outcome not } \\
\text { included in review }\end{array}$ \\
\hline Hale, AR & $\begin{array}{l}\text { Perioperative medication management: expanding the role of the preadmission clinic pharmacist } \\
\text { in a single centre, randomised controlled trial of collaborative prescribing }\end{array}$ & 2013 & $\begin{array}{l}\text { Outcome not } \\
\text { included in review }\end{array}$ \\
\hline Haq, N & $\begin{array}{l}\text { Impact of pharmacists-led intervention programme towards knowledge, attitude and practice } \\
\text { among hepatitis B patients in Pakistan: a nonclinical randomized controlled trial }\end{array}$ & 2013 & $\begin{array}{l}\text { Meeting report or } \\
\text { editorial }\end{array}$ \\
\hline Holland, R & $\begin{array}{l}\text { Delivering a home-based medication review: process measures from the HOMER randomised } \\
\text { controlled trial }\end{array}$ & 2006 & $\begin{array}{l}\text { Outcome not } \\
\text { included in review }\end{array}$ \\
\hline Holmes-Rovner, M & $\begin{array}{l}\text { Does outpatient telephone coaching add to hospital quality improvement following hospitalization } \\
\text { for acute coronary syndrome? }\end{array}$ & 2008 & $\begin{array}{l}\text { No pharmacist } \\
\text { intervention }\end{array}$ \\
\hline Huang, A & The medication reconciliation process: keys to success & 2012 & $\begin{array}{l}\text { Meeting report or } \\
\text { editorial }\end{array}$ \\
\hline Israel, EN & Underutilization of cardiovascular medications: effect of a continuity-of-care program & 2013 & \begin{tabular}{|l|} 
Outcome not \\
included in review
\end{tabular} \\
\hline Khdour, MR & Clinical pharmacy-led disease and medicine management programme for patients with COPD & 2009 & No transitional care \\
\hline Kimball, S & $\begin{array}{l}\text { Testing a teaching appointment and geragogy-based approach to medication knowledge at dis- } \\
\text { charge }\end{array}$ & 2010 & Study design \\
\hline Kripalani, S & $\begin{array}{l}\text { Pharmacist intervention for low literacy in cardiovascular disease (PILL-CVD): a randomized con- } \\
\text { trolled trial }\end{array}$ & 2011 & $\begin{array}{l}\text { Meeting report or } \\
\text { editorial }\end{array}$ \\
\hline Kucukarslan, SN & $\begin{array}{l}\text { Pharmacists on rounding teams reduce preventable adverse drug events in hospital general medi- } \\
\text { cine units }\end{array}$ & 2003 & Study design \\
\hline Kwan, Y & Pharmacist medication assessments in a surgical preadmission clinic & 2007 & \begin{tabular}{l|} 
Outcome not \\
included in review
\end{tabular} \\
\hline Lalonde, $\mathrm{L}$ & $\begin{array}{l}\text { Effectiveness of a medication discharge plan for transitions of care from hospital to outpatient } \\
\text { settings }\end{array}$ & 2008 & \begin{tabular}{|l|} 
Outcome not \\
included in review
\end{tabular} \\
\hline Laramee, AS & $\begin{array}{l}\text { Case management in a heterogeneous congestive heart failure population: a randomized con- } \\
\text { trolled trial }\end{array}$ & 2003 & $\begin{array}{l}\text { No pharmacist } \\
\text { intervention }\end{array}$ \\
\hline Linne, $\mathrm{AB}$ & $\begin{array}{l}\text { Effects of systematic education on heart failure patients' knowledge after } 6 \text { months: a randomised } \\
\text { controlled trial }\end{array}$ & 1999 & $\begin{array}{l}\text { Outcome not } \\
\text { included in review }\end{array}$ \\
\hline Lowe, $\mathrm{CJ}$ & $\begin{array}{l}\text { Effects of self-medication programme on knowledge of drugs and compliance with treatment in } \\
\text { elderly patients }\end{array}$ & 1995 & $\begin{array}{l}\text { Outcome not } \\
\text { included in review }\end{array}$ \\
\hline Mannheimer, B & Drug-related problems and pharmacotherapeutic advisory intervention at a medicine clinic & 2006 & $\begin{array}{l}\text { No pharmacist } \\
\text { intervention }\end{array}$ \\
\hline Marotti, SB & $\begin{array}{l}\text { A randomised controlled trial of pharmacist medication histories and supplementary prescribing } \\
\text { on medication errors in postoperative medications }\end{array}$ & 2011 & $\begin{array}{l}\text { Outcome not } \\
\text { included in review }\end{array}$ \\
\hline Marusic, S & The effect of pharmacotherapeutic counseling on readmissions and emergency department visits & 2013 & $\begin{array}{l}\text { No pharmacist } \\
\text { intervention }\end{array}$ \\
\hline Menditto, E & A ten years longer life: a therapeutic education program for hypertensive patients & 2012 & $\begin{array}{l}\text { Meeting report or } \\
\text { editorial }\end{array}$ \\
\hline Muniz, J & $\begin{array}{l}\text { The effect of post-discharge educational intervention on patients in achieving objectives in modi- } \\
\text { fiable risk factors six months after discharge following an episode of acute coronary syndrome, } \\
\text { (CAM-2 Project): a randomized controlled trial }\end{array}$ & 2010 & $\begin{array}{l}\text { No pharmacist } \\
\text { intervention }\end{array}$ \\
\hline Murray, MD & $\begin{array}{l}\text { Effect of a pharmacist on adverse drug events and medication errors in outpatients with cardio- } \\
\text { vascular disease }\end{array}$ & 2009 & No transitional care \\
\hline Nickerson, A & $\begin{array}{l}\text { Drug-therapy problems, inconsistencies and omissions identified during a medication reconcilia- } \\
\text { tion and seamless care service }\end{array}$ & 2005 & \begin{tabular}{|l|} 
Outcome not \\
included in review
\end{tabular} \\
\hline O'Connor, M & $\begin{array}{l}\text { Prevention of adverse drug events in hospitalized older patients: a randomised controlled trial } \\
\text { using STOPP/START criteria }\end{array}$ & 2012 & $\begin{array}{l}\text { Meeting report or } \\
\text { editorial }\end{array}$ \\
\hline Olson, KL & Outcomes of patients discharged from pharmacy-managed cardiovascular disease management & 2009 & No transitional care \\
\hline Owens, NJ & $\begin{array}{l}\text { The senior care study: the relationship between optimal pharmacotherapy and patient mental } \\
\text { status }\end{array}$ & 1990 & $\begin{array}{l}\text { Meeting report or } \\
\text { editorial }\end{array}$ \\
\hline Pacini, $\mathrm{M}$ & Home-based medication review in older people: is it cost effective? & 2007 & $\begin{array}{l}\text { Outcome not } \\
\text { included in review }\end{array}$ \\
\hline
\end{tabular}




\begin{tabular}{|c|c|c|c|}
\hline Primary Author & Primary Title & $\begin{array}{c}\text { Publication } \\
\text { Year }\end{array}$ & $\begin{array}{l}\text { Reason for } \\
\text { Exclusion }\end{array}$ \\
\hline Pai, AB & $\begin{array}{l}\text { Reduced drug use and hospitalization rates in patients undergoing hemodialysis who received } \\
\text { pharmaceutical care: a 2-year, randomized, controlled study }\end{array}$ & 2009 & No transitional care \\
\hline Parry, C & $\begin{array}{l}\text { The care transitions intervention: a patient-centered approach to ensuring effective transfers } \\
\text { between sites of geriatric care }\end{array}$ & 2003 & $\begin{array}{l}\text { No pharmacist } \\
\text { intervention }\end{array}$ \\
\hline Perera, KY & $\begin{array}{l}\text { Medium of language in discharge summaries: would the use of native language improve patients' } \\
\text { knowledge of their illness and medications? }\end{array}$ & 2012 & $\begin{array}{l}\text { No pharmacist } \\
\text { intervention }\end{array}$ \\
\hline Peterson, GM & Impact of pharmacist-conducted home visits on the outcomes of lipid-lowering drug therapy & 2004 & $\begin{array}{l}\text { Targets specific } \\
\text { drug }\end{array}$ \\
\hline Pitner, J & Specialty geriatric evaluation and management teams reduce adverse drug reactions & 2004 & $\begin{array}{l}\text { Meeting report or } \\
\text { editorial }\end{array}$ \\
\hline Polack, J & $\begin{array}{l}\text { Evaluation of different methods of providing medication-related education to patients following } \\
\text { myocardial infarction }\end{array}$ & 2008 & $\begin{array}{l}\text { Outcome not } \\
\text { included in review }\end{array}$ \\
\hline Raynor, DK & Effects of computer generated reminder charts on patients' compliance with drug regimens & 1993 & $\begin{array}{l}\text { Outcome not } \\
\text { included in review }\end{array}$ \\
\hline Rice, KL & $\begin{array}{l}\text { Disease management program for chronic obstructive pulmonary disease: a randomized con- } \\
\text { trolled trial }\end{array}$ & 2010 & $\begin{array}{l}\text { No pharmacist } \\
\text { intervention }\end{array}$ \\
\hline Rich, MW & $\begin{array}{l}\text { Effect of a multidisciplinary intervention on medication compliance in elderly patients with con- } \\
\text { gestive heart failure }\end{array}$ & 1996 & $\begin{array}{l}\text { No pharmacist } \\
\text { intervention }\end{array}$ \\
\hline Rienstra, M & A specialized atrial fibrillation clinic: Improving care and costs for patients with atrial fibrillation & 2013 & $\begin{array}{l}\text { Meeting report or } \\
\text { editorial }\end{array}$ \\
\hline Robinson, A & Guided self-management and patient-directed follow-up of ulcerative colitis: a randomised trial & 2001 & $\begin{array}{l}\text { No pharmacist } \\
\text { intervention }\end{array}$ \\
\hline Sadik, A & Pharmaceutical care of patients with heart failure & 2005 & No transitional care \\
\hline Salanitro, A & $\begin{array}{l}\text { Factors associated with admission and discharge medication reconciliation errors at } 2 \text { teaching } \\
\text { hospitals }\end{array}$ & 2011 & Study design \\
\hline Salanitro, AH & Effect of patient- and medication-related factors on inpatient medication reconciliation errors & 2012 & Study design \\
\hline Saleem, F & $\begin{array}{l}\text { A non-clinical randomized controlled trial assessing impact of pharmacists led intervention pro- } \\
\text { gramme for enhancing medication adherence and health-related quality of life }\end{array}$ & 2012 & $\begin{array}{l}\text { Meeting report or } \\
\text { editorial }\end{array}$ \\
\hline Sanchez Ulayar, A & $\begin{array}{l}\text { Pharmaceutical intervention upon hospital discharge to strengthen understanding and adherence } \\
\text { to pharmacological treatment }\end{array}$ & 2012 & $\begin{array}{l}\text { Full-text not in } \\
\text { English }\end{array}$ \\
\hline Schwaab, B & $\begin{array}{l}\text { In-patient cardiac rehabilitation versus medical care - a prospective multicentre controlled } 12 \\
\text { months follow-up in patients with coronary heart disease }\end{array}$ & 2011 & $\begin{array}{l}\text { No pharmacist } \\
\text { intervention }\end{array}$ \\
\hline Shah, M & Diabetes transitional care from inpatient to outpatient setting: pharmacist discharge counseling & 2013 & $\begin{array}{l}\text { Outcome not } \\
\text { included in review }\end{array}$ \\
\hline Simpson, TRG & $\begin{array}{l}\text { A comprehensive case management programme to prevent chronic obstructive pulmonary dis- } \\
\text { ease hospitalisations }\end{array}$ & 2013 & $\begin{array}{l}\text { Meeting report or } \\
\text { editorial }\end{array}$ \\
\hline Smith, L & $\begin{array}{l}\text { An investigation of hospital generated pharmaceutical care when patients are discharged home } \\
\text { from hospital }\end{array}$ & 1997 & $\begin{array}{l}\text { Outcome not } \\
\text { included in review }\end{array}$ \\
\hline Stamatakis, MK & Effectiveness of a pharmacist-initiated continuity of care program for chronic dialysis patients & 1998 & Study design \\
\hline Stewart, S & $\begin{array}{l}\text { Home-based management for chronic heart failure reduces recurrent hospital stay and total } \\
\text { healthcare costs compared with a clinic-based program: results from the WHICH? Trial }\end{array}$ & 2012 & Study design \\
\hline Stowasser, DA & $\begin{array}{l}\text { A randomised controlled trial of medication liaison services - acceptance and use by health pro- } \\
\text { fessionals }\end{array}$ & 2002 & $\begin{array}{l}\text { Outcome not } \\
\text { included in review }\end{array}$ \\
\hline Talasaz, AH & The potential role of clinical pharmacy services in patients with cardiovascular diseases & 2012 & Study design \\
\hline Tompson, AJ & $\begin{array}{l}\text { Utilizing community pharmacy dispensing records to disclose errors in hospital admission drug } \\
\text { charts }\end{array}$ & 2012 & $\begin{array}{l}\text { Outcome not } \\
\text { included in review }\end{array}$ \\
\hline Tsuyuki, RT & A multicenter disease management program for hospitalized patients with heart failure & 2004 & $\begin{array}{l}\text { Mo pharmacist } \\
\text { intervention }\end{array}$ \\
\hline Ulrik, CS & $\begin{array}{l}\text { No benefit and potential harm with an educational and care management programme for chronic } \\
\text { obstructive pulmonary disease }\end{array}$ & 2013 & $\begin{array}{l}\text { Meeting report or } \\
\text { editorial }\end{array}$ \\
\hline Van der Linden, L & $\begin{array}{l}\text { Reduction of polypharmacy in geriatric inpatients using the RASP list: a cluster-randomized con- } \\
\text { trolled trial }\end{array}$ & 2013 & $\begin{array}{l}\text { Meeting report or } \\
\text { editorial }\end{array}$ \\
\hline Vuong, $\mathrm{T}$ & Implementation of a community liaison pharmacy service: a randomised controlled trial & 2008 & $\begin{array}{l}\text { Outcome not } \\
\text { included in review }\end{array}$ \\
\hline Wei, L & $\begin{array}{l}\text { Effect of pharmaceutical care on medication adherence and hospital admission in patients with } \\
\text { chronic obstructive pulmonary disease (COPD): a randomized controlled study }\end{array}$ & 2014 & No transitional care \\
\hline Williams, JB & $\begin{array}{l}\text { Secondary prevention after coronary artery bypass graft surgery: findings of a national random- } \\
\text { ized controlled trial and sustained society-led incorporation into practice }\end{array}$ & 2011 & $\begin{array}{l}\text { No pharmacist } \\
\text { intervention }\end{array}$ \\
\hline
\end{tabular}


APPENDIX F Reason for Excluding Articles After Full-Text Review (continued)

\begin{tabular}{|c|c|c|c|}
\hline Primary Author & Primary Title & $\begin{array}{l}\text { Publication } \\
\quad \text { Year }\end{array}$ & $\begin{array}{l}\text { Reason for } \\
\text { Exclusion }\end{array}$ \\
\hline Williams, M & Project impact: Improving patient adherence through communication at transition & 2013 & $\begin{array}{l}\text { Meeting report or } \\
\text { editorial }\end{array}$ \\
\hline Williford, SL & Impact of pharmacist counseling on medication knowledge and compliance & 1995 & $\begin{array}{l}\text { Outcome not } \\
\text { included in review }\end{array}$ \\
\hline Willoch, K & Handling drug-related problems in rehabilitation patients: a randomized study & 2012 & No transitional care \\
\hline$\overline{\mathrm{Wu}, \mathrm{JR}}$ & $\begin{array}{l}\text { Effect of a medication-taking behavior feedback theory-based intervention on outcomes in } \\
\text { patients with heart failure }\end{array}$ & 2012 & $\begin{array}{l}\text { No pharmacist } \\
\text { intervention }\end{array}$ \\
\hline Zerafa, N & $\begin{array}{l}\text { Impact of drugs counselling by an undergraduate pharmacist on cardiac surgical patient's com- } \\
\text { pliance to medicines }\end{array}$ & 2011 & $\begin{array}{l}\text { Outcome not } \\
\text { included in review }\end{array}$ \\
\hline Zermansky, AG & $\begin{array}{l}\text { Clinical medication review by a pharmacist of patients on repeat prescriptions in general prac- } \\
\text { tice: A randomised controlled trial }\end{array}$ & 2002 & No transitional care \\
\hline Zhao, Y & $\begin{array}{l}\text { Effects of a postdischarge transitional care programme for patients with coronary heart disease in } \\
\text { China: a randomised controlled trial }\end{array}$ & 2009 & $\begin{array}{l}\text { No pharmacist } \\
\text { intervention }\end{array}$ \\
\hline
\end{tabular}



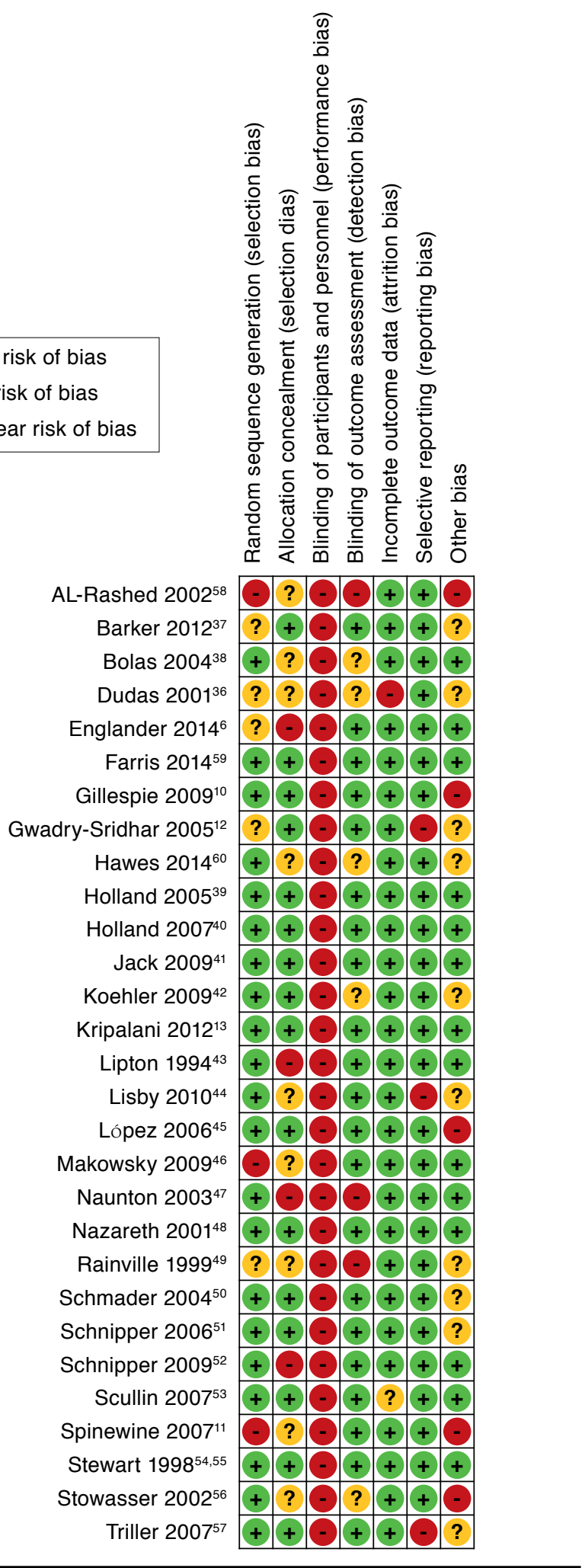


\section{APPENDIX H Cross-Tab Intervention Versus Outcome Measurement}

Outcome Measurement

Pharmacist Intervention

1. Admission reconciliation

2. Patient counseling at admission

3. Pharmacist is part of medical team

4. Medication review

5. Patient counseling during admission

6. Discharge reconciliation

7. Patient counseling at discharge

8. Patient discharge letter

9. Transmission

10. Patient-centered follow-up

11. HCP-centered follow-up

12. Extra postdischarge follow-up

13. Tailored intervention

14. Provision of adherence aides

15. Dispensing/logistics aides

Sum column

\begin{tabular}{|c|c|c|c|c|c|c|c}
\hline \multicolumn{7}{|c|}{ Outcome Measurement } \\
\hline Mortality & Readmissions & $\begin{array}{c}\text { ED } \\
\text { Visits }\end{array}$ & $\begin{array}{c}\text { Composite } \\
\text { RE }\end{array}$ & $\begin{array}{c}\text { Composite } \\
\text { RM }\end{array}$ & $\begin{array}{c}\text { Composite } \\
\text { REM }\end{array}$ & ADEs & Sum Row \\
\hline 5 & 7 & 4 & 3 & - & - & 3 & 22 \\
\hline 2 & 3 & 1 & 1 & - & - & - & 7 \\
\hline 2 & 4 & 2 & 1 & - & 1 & - & 10 \\
\hline 5 & 7 & 4 & 4 & - & 2 & 2 & 24 \\
\hline 3 & 6 & 3 & 2 & - & 2 & 2 & 18 \\
\hline 4 & 8 & 2 & 2 & - & - & 1 & 17 \\
\hline 5 & 10 & 3 & 3 & - & - & 2 & 23 \\
\hline 3 & 6 & 2 & 1 & - & 1 & 2 & 15 \\
\hline 5 & 8 & 3 & 2 & - & - & 1 & 19 \\
\hline 10 & 16 & 7 & 5 & 2 & 1 & 2 & 43 \\
\hline 8 & 12 & 5 & 2 & 2 & - & 2 & 31 \\
\hline 5 & 8 & 1 & - & - & 1 & 1 & 16 \\
\hline 5 & 5 & 3 & 1 & 2 & - & 1 & 17 \\
\hline 3 & 5 & 3 & - & 2 & - & 2 & 15 \\
\hline 5 & 6 & 1 & - & - & - & - & 12 \\
\hline 70 & 11 & 44 & 27 & 8 & 8 & 21 & \\
\hline
\end{tabular}

$A D E s=$ adverse drug events; $E D=$ emergency department; $H C P=$ health care provider; $R E=$ readmissions and ED visits; $R E M=$ readmissions, $E D$ visits, and mortality; $R M=$ readmissions and mortality. 
Identifying the Optimal Role for Pharmacists in Care Transitions: A Systematic Review

\section{APPENDIX I Categorization of Interventions Performed by Other Health Care Providers}

\begin{tabular}{|c|c|c|c|}
\hline Author & HCP Type & Performed Intervention & Categorization \\
\hline Barker et al., $2012^{37}$ & PCP & $\begin{array}{l}\text { Assessment of drug-related recommendations identified by pharmacists' postdischarge } \\
\text { follow-ups and acting upon them if deemed necessary }\end{array}$ & $\begin{array}{l}\text { Act on drug-related } \\
\text { recommendations (PD) }\end{array}$ \\
\hline Dudas et al., $2001^{36}$ & PCP & $\begin{array}{l}\text { Assessment of drug-related recommendations identified by pharmacists' postdischarge } \\
\text { follow-ups and acting upon them if deemed necessary }\end{array}$ & $\begin{array}{l}\text { Act on drug-related } \\
\text { recommendations (PD) }\end{array}$ \\
\hline Englander et al., 20146 & $\mathrm{~N}$ & $\begin{array}{l}\text { Coaching and education, including postdischarge phone calls and home visits for } \\
\text { highest risk patients }\end{array}$ & $\begin{array}{l}\text { Coordination, counseling, } \\
\text { risk assessment }\end{array}$ \\
\hline Farris et al., 201459 & PCP & $\begin{array}{l}\text { Assessment of drug-related recommendations identified by pharmacists' postdischarge } \\
\text { follow-ups and acting upon them if deemed necessary }\end{array}$ & $\begin{array}{l}\text { Act on drug-related } \\
\text { recommendations (PD) }\end{array}$ \\
\hline Gillespie et al., 200910 & S & $\begin{array}{l}\text { Assessment of drug-related recommendations identified by pharmacists' clinical medi- } \\
\text { cation reviews and acting upon them if deemed necessary }\end{array}$ & $\begin{array}{l}\text { Act on drug-related } \\
\text { recommendations (DA) }\end{array}$ \\
\hline $\begin{array}{l}\text { Gwadry-Sridhar et al., } \\
2005^{12}\end{array}$ & $\mathrm{~N}$ & Patient counseling is a team intervention with pharmacist & Counseling \\
\hline Holland et al., $2005^{39}$ & PCP & $\begin{array}{l}\text { Assessment of drug-related recommendations identified by pharmacists' postdischarge } \\
\text { follow-ups and acting upon them if deemed necessary }\end{array}$ & $\begin{array}{l}\text { Act on drug-related } \\
\text { recommendations (PD) }\end{array}$ \\
\hline Holland et al., $2007^{40}$ & PCP & $\begin{array}{l}\text { Assessment of drug-related recommendations identified by pharmacists' postdischarge } \\
\text { follow-ups and acting upon them if deemed necessary }\end{array}$ & $\begin{array}{l}\text { Act on drug-related } \\
\text { recommendations (PD) }\end{array}$ \\
\hline \multirow[t]{2}{*}{ Jack et al., $2009^{41}$} & $\mathrm{~N}$ & Coordinate discharge plan, educate, and prepare patients for discharge & Coordination, counseling \\
\hline & PCP & $\begin{array}{l}\text { Assessment of drug-related recommendations identified by pharmacists' postdischarge } \\
\text { follow-ups and acting upon them if deemed necessary }\end{array}$ & $\begin{array}{l}\text { Act on drug-related } \\
\text { recommendations (PD) }\end{array}$ \\
\hline \multirow[t]{2}{*}{ Koehler et al., $2009^{42}$} & SN & $\begin{array}{l}\text { Daily condition specific education, identify and address discharge barriers, self-manage- } \\
\text { ment discharge teaching, follow-up call at 5-7 days postdischarge }\end{array}$ & Counseling \\
\hline & S & $\begin{array}{l}\text { Assessment of drug-related recommendations identified by pharmacists' clinical medi- } \\
\text { cation reviews and acting upon them if deemed necessary }\end{array}$ & $\begin{array}{l}\text { Act on drug-related } \\
\text { recommendations (DA) }\end{array}$ \\
\hline \multirow[t]{2}{*}{ Kripalani et al., $2012^{13}$} & SW & Assistance obtaining discharge medications & Coordination \\
\hline & PCP & $\begin{array}{l}\text { Assessment of drug-related recommendations identified by pharmacists' postdischarge } \\
\text { follow-ups and acting upon them if deemed necessary }\end{array}$ & $\begin{array}{l}\text { Act on drug-related } \\
\text { recommendations (PD) }\end{array}$ \\
\hline Lipton and Bird, 199443 & PCP & $\begin{array}{l}\text { Assessment of drug-related recommendations identified by pharmacists' postdischarge } \\
\text { follow-ups and acting upon them if deemed necessary }\end{array}$ & $\begin{array}{l}\text { Act on drug-related } \\
\text { recommendations (PD) }\end{array}$ \\
\hline Makowsky et al., 200946 & S & $\begin{array}{l}\text { Assessment of drug-related recommendations identified by pharmacists' clinical medi- } \\
\text { cation reviews and acting upon them if deemed necessary }\end{array}$ & $\begin{array}{l}\text { Act on drug-related } \\
\text { recommendations (DA) }\end{array}$ \\
\hline Naunton et al., $2003^{47}$ & PCP & $\begin{array}{l}\text { Assessment of drug-related recommendations identified by pharmacists' postdischarge } \\
\text { follow-ups and acting upon them if deemed necessary }\end{array}$ & $\begin{array}{l}\text { Act on drug-related } \\
\text { recommendations (PD) }\end{array}$ \\
\hline Nazareth et al., $2001^{48}$ & PCP & $\begin{array}{l}\text { Assessment of drug-related recommendations identified by pharmacists' postdischarge } \\
\text { follow-ups and acting upon them if deemed necessary }\end{array}$ & $\begin{array}{l}\text { Act on drug-related } \\
\text { recommendations (PD) }\end{array}$ \\
\hline \multirow[t]{2}{*}{ Rainville, $1999^{49}$} & $\mathrm{SN}$ & Identify patients with potential rehospitalization risks and determined corrective action & Risk assessment and referral \\
\hline & S & $\begin{array}{l}\text { Assessment of drug-related recommendations identified by pharmacists' clinical medi- } \\
\text { cation reviews and acting upon them if deemed necessary }\end{array}$ & $\begin{array}{l}\text { Act on drug-related } \\
\text { recommendations (DA) }\end{array}$ \\
\hline Schmader et al., 200450 & S & $\begin{array}{l}\text { Assessment of drug-related recommendations identified by pharmacists' clinical medi- } \\
\text { cation reviews and acting upon them if deemed necessary }\end{array}$ & $\begin{array}{l}\text { Act on drug-related } \\
\text { recommendations (DA) }\end{array}$ \\
\hline \multirow[t]{2}{*}{ Schnipper et al., $2006^{51}$} & S & $\begin{array}{l}\text { Assessment of drug-related recommendations identified by pharmacists' clinical medi- } \\
\text { cation reviews and acting upon them if deemed necessary }\end{array}$ & $\begin{array}{l}\text { Act on drug-related } \\
\text { recommendations (DA) }\end{array}$ \\
\hline & PCP & $\begin{array}{l}\text { Assessment of drug-related recommendations identified by pharmacists' postdischarge } \\
\text { follow-ups and acting upon them if deemed necessary }\end{array}$ & $\begin{array}{l}\text { Act on drug-related } \\
\text { recommendations (PD) }\end{array}$ \\
\hline \multirow[t]{2}{*}{ Schnipper et al., 200952} & S & $\begin{array}{l}\text { 1. Taking preadmission medication histories (PMH), referring to PMH at discharge } \\
\text { 2. Assessment of drug-related recommendations identified by pharmacists' clinical } \\
\text { medication reviews and acting upon them if deemed necessary }\end{array}$ & $\begin{array}{l}\text { 1. Reconciliation } \\
\text { 2. Act on drug-related } \\
\text { recommendations (DA) }\end{array}$ \\
\hline & $\mathrm{N}$ & Performing discharge counseling & Counseling \\
\hline Scullin et al., $2007^{53}$ & S & $\begin{array}{l}\text { Assessment of drug-related recommendations identified by pharmacists' clinical medi- } \\
\text { cation reviews and acting upon them if deemed necessary }\end{array}$ & $\begin{array}{l}\text { Act on drug-related } \\
\text { recommendations (DA) }\end{array}$ \\
\hline Stewart et al., $1998^{54}$ & $\mathrm{~N}$ & $\begin{array}{l}\text { Predischarge patient treatment adherence counseling, report clinical deterioration. } \\
\text { At home visit: detect clinical deterioration or adverse drug events and referral to GP }\end{array}$ & $\begin{array}{l}\text { Counseling, risk } \\
\text { assessment, and referral }\end{array}$ \\
\hline Stewart et al., $1998^{55}$ & $\mathrm{~N}$ & $\begin{array}{l}\text { Predischarge patient treatment adherence counseling, report clinical deterioration. } \\
\text { At home visit: detect clinical deterioration or adverse drug events and referral to GP }\end{array}$ & $\begin{array}{l}\text { Counseling, risk } \\
\text { assessment, and referral }\end{array}$ \\
\hline Stowasser et al., $2002^{56}$ & GP, CP & Confirmation medication history at admission & Verification \\
\hline Triller et al., 200757 & $\mathrm{~S}$ & $\begin{array}{l}\text { Assessment of drug-related recommendations identified by pharmacists' postdischarge } \\
\text { follow-ups and acting upon them if deemed necessary }\end{array}$ & $\begin{array}{l}\text { Act on drug-related } \\
\text { recommendations (PD) }\end{array}$ \\
\hline
\end{tabular}

$C P=$ community pharmacist $D A=$ during admission; $G P=$ general practitioner; $N=$ nurse; $P C P=$ primary care provider; $P D=$ postdischarge; $S=$ specialist; $S N=$ specialized nurse; $S W=$ social worker. 


\section{APPENDIX J Categorization of Interventions Performed Per Included Study}

\begin{tabular}{|c|c|c|c|c|c|c|c|c|c|c|c|c|c|c|c|}
\hline & \multicolumn{15}{|c|}{ Pharmacist Intervention ${ }^{a}$} \\
\hline Study Author/Date & 1 & 2 & 3 & $4^{b}$ & 5 & 6 & 7 & 8 & 9 & $10^{\mathrm{c}}$ & 11 & 12 & 13 & 14 & 15 \\
\hline Al-Rashed et al., $2002^{58}$ & & & & & & & $\bullet$ & & & & & & & 0 & \\
\hline Barker et al., $2012^{37}$ & & & & & & & & & & $\mathbf{H}$ & $\bullet$ & 0 & & & - \\
\hline Bolas et al., $2004^{38}$ & ○ & ○ & & & ○ & 0 & $\bullet$ & 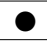 & $\bullet$ & & & & & & - \\
\hline Dudas et al., $2001^{36}$ & & & & & & & & & & $\mathbf{T}$ & - & & & & \\
\hline Englander et al., 20146 & & & & 1 & - & - & & & & & & & & & - \\
\hline Farris et al., 201459 & 0 & & & & 0 & $\bullet$ & $\bullet$ & $\bullet$ & $\bullet$ & $\mathbf{T}$ & $\bullet$ & $\bullet$ & & 0 & \\
\hline Gillespie et al., $2009^{10}$ & 0 & 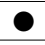 & 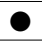 & 3 & 0 & & $\bullet$ & $\bullet$ & $\bullet$ & $\mathbf{T}$ & & & $\bullet$ & & \\
\hline Gwadry-Sridhar et al., $2005^{12}$ & & & & 2 & ○ & & & $\bullet$ & & & & & & & \\
\hline Hawes et al., 201460 & & & & & & & & & & C & & & & & \\
\hline Holland et al., $2005^{39}$ & & & & & & & & & & $\mathbf{H}$ & $\bullet$ & $\bullet$ & & & $\bullet$ \\
\hline Holland et al., $2007^{40}$ & & & & & & & & & & $\mathbf{H}$ & $\bullet$ & 0 & & & $\bullet$ \\
\hline Jack et al., $2009^{41}$ & & & & & & & & & & $\mathbf{T}$ & $\bullet$ & & & & \\
\hline Koehler et al., $2009^{42}$ & 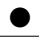 & & & 3 & ○ & $\bullet$ & $\bullet$ & & $\bullet$ & $\mathbf{T}$ & & & & & \\
\hline Kripalani et al., $2012^{13}$ & 0 & & & 2 & 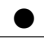 & & $\bullet$ & $\bullet$ & & $\mathbf{T}$ & $\bullet$ & & 0 & $\bullet$ & \\
\hline Lipton and Bird, 199443 & & & & & & & $\bullet$ & & & $\mathbf{T}$ & $\bullet$ & 0 & & & \\
\hline Lisby et al., $2010^{44}$ & 0 & & & 1 & & & & & & & & & & & \\
\hline López Cabezas et al., $2006^{45}$ & & & & & & & $\bullet$ & & & $\mathbf{T}$ & & & & & \\
\hline Makowsky et al., $2009^{46}$ & 0 & & 0 & 3 & & $\bullet$ & $\bullet$ & $\bullet$ & $\bullet$ & & & & & & \\
\hline Naunton et al., $2003^{47}$ & & & & & & & & & & $\mathbf{H}$ & $\bullet$ & & & $\bullet$ & \\
\hline Nazareth et al., $2001^{48}$ & & & & & & $\bullet$ & $\bullet$ & $\bullet$ & $\bullet$ & $\mathbf{H}$ & $\bullet$ & & 0 & & \\
\hline Rainville, $1999^{49}$ & & & 0 & 3 & 0 & & & & & $\mathbf{T}$ & & $\bullet$ & & & \\
\hline Schmader et al., 200450 & & & 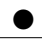 & 3 & & & & & & & & & & & \\
\hline Schnipper et al., $2006^{51}$ & & & & 3 & & $\bullet$ & $\bullet$ & & & $\mathbf{T}$ & 0 & & & & \\
\hline Schnipper et al., $2009^{52}$ & 0 & & & 3 & & & & & & & & & & & \\
\hline Scullin et al., $2007^{53}$ & $\bullet$ & 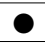 & & 3 & ○ & $\bullet$ & - & - & $\bullet$ & & & & $\bullet$ & & $\bullet$ \\
\hline Spinewine et al., $2007^{11}$ & 0 & & 0 & $\mathbf{1}$ & & & $\bullet$ & & $\bullet$ & & & & & & \\
\hline Stewart et al., $1998^{54}$ & & & & & & & & & & $\mathbf{H}$ & 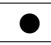 & & 0 & $\bullet$ & \\
\hline Stewart et al., $1998^{55}$ & & & & & & & & & & $\mathbf{H}$ & 0 & & 0 & - & \\
\hline Stowasser et al., $2002^{56}$ & - & & & & & - & & & - & & & & & & \\
\hline Triller et al., $2007^{57}$ & & & & & & & & & & $\mathbf{H}$ & - & - & & & \\
\hline
\end{tabular}

aPharmacist intervention; number according to the pharmacist intervention model (Table 1).

${ }^{b}$ Medication review levels: 1 = prescription review, $2=$ adherence support review (with patient present), $3=$ clinical review, $4=$ clinical review with prescribing. cPatient-centered follow-up: $C=$ clinic visit, $H=$ home visit, $T=$ telephone call. 